\title{
CELL-TO-MUSCLE HOMOGENIZATION. APPLICATION TO A CONSTITUTIVE LAW FOR THE MYOCARDIUM*
}

\author{
Denis Caillerie ${ }^{1}$, Ayman Mourad ${ }^{1,2}$ And Annie Raoult ${ }^{2,3}$
}

\begin{abstract}
We derive a constitutive law for the myocardium from the description of both the geometrical arrangement of cardiomyocytes and their individual mechanical behaviour. We model a set of cardiomyocytes by a quasiperiodic discrete lattice of elastic bars interacting by means of moments. We work in a large displacement framework and we use a discrete homogenization technique. The macroscopic constitutive law is obtained through the resolution of a nonlinear self-equilibrum system of the discrete lattice reference cell.
\end{abstract}

Mathematics Subject Classification. 74L15, 74Q05, 74Q15, 92B05.

\section{INTRODUCTION}

Cardiac modelling is a field of rapid progress. Better knowledge of the heart anatomy and of the heart functions is expected from today advances in accuracy of experimental data, in functional imaging, in mechanical understanding, and in numerical simulation.

The myocardium is never at rest and its overall deformation results from the individual deformation of the cardiomyocytes, or more precisely of their contractile units. From an anatomic point of view, recent work has been devoted to better describe the geometrical arrangement of cardiomyocytes and of cardiac fibres. Streeter [23], for instance, conjectured that cardiac fibres follow geodesic paths on a nested set of toroidal surfaces. Such an organization, which is specific to the myocardium, should probably be taken into account in a complete geometrical and mechanical heart modelling. Let us mention that this hypothesis was investigated from a theoretical point of view by Peskin [20], who examined its validity in models containing a design constraint on possible stress-strain laws. In [18], we were interested in checking Streeter's conjecture against experimental data on the fibre orientation obtained in polarized light microscopy by Jouk et al. [13].

From a mechanical point of view, several authors contributed in the derivation of constitutive laws for the myocardium. Obviously, experimentation is difficult and the activation process adds to the modelling complexity. We refer the reader to works by Arts et al. [1], Cai [5], Chadwick [7], Chapelle et al. [8], Fung [11], Humphrey et al. [14,15], Lin and Yin [16], Nash and Hunter [19], Taber and Perucchio [24], Usyk et al. [27], Yin et al. [29] among many others and to references therein. The approach is generally phenomenological. A basic way of

Keywords and phrases. Myocardium, constitutive law, homogenization, large deformations.

* This work was supported by the Fonds National de la Science (ACI Modélisation mathématique, mécanique et numérique du myocarde) and by the Région Rhône-Alpes (Projet Mathématiques pour ADéMo).

${ }^{1}$ Laboratoire Sols, Solides, Structures, BP 53, 38041 Grenoble Cedex 9, France. e-mail: Denis.Caillerie@inpg.fr

2 Laboratoire de Modélisation et Calcul, BP 53, 38041 Grenoble Cedex 9, France. e-mail: Ayman.Mourad@imag.fr

3 Laboratoire TIMC, Domaine de la Merci, 38076 La Tronche Cedex, France. e-mail: Annie.Raoult@imag.fr 

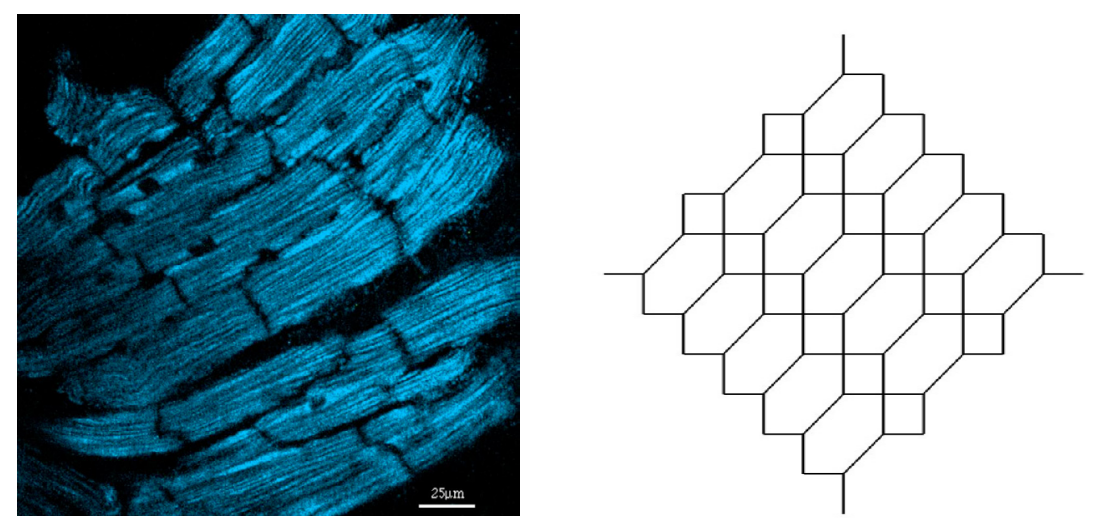

Figure 1. Myocardial cells joined by anastomoses (left). A bidimensional lattice (right).

modelling consists in assuming that the stress tensor $\boldsymbol{\sigma}$ reads as the sum $\boldsymbol{\sigma}=-p \boldsymbol{I}+T \boldsymbol{\tau} \otimes \boldsymbol{\tau}+\boldsymbol{\sigma}_{\boldsymbol{p}}$ of an active tensor $T \boldsymbol{\tau} \otimes \boldsymbol{\tau}$ where $\boldsymbol{\tau}$ denotes the unit vector of the fibre direction and where $T$ is the active tension, of a passive stress tensor $\boldsymbol{\sigma}_{\boldsymbol{p}}$, and of a term due to the incompressibility. Elastic energies can be used as well. Some authors postulate that the myocardium is a hyperelastic homogeneous material. Isotropic energies were first considered, then extended to more realistic orthotropic functions. Experiments that are necessary to fit the material constraints can be performed either on the passive myocardium, or in a contracted state.

We propose to follow a different approach and we aim at deriving a global - or macroscopic - constitutive law for the myocardium from microscopic mechanical models. By microscopic level, we mean the cardiac cell level. Cardiac cells, or cardiomyocytes, are small, 60-100 $\mathrm{m}$ long, cylindrical structures. In an adult myocardium, the left ventricle is typically $8 \mathrm{~cm}$ long with a $5 \mathrm{~cm}$ inner diameter. Cardiomyocytes are connected into a network by anastomoses making I-junctions or Y-junctions, see Figure 1. This specific organization drastically differs from the cell organization into a skeletal muscle, where a fibre can consist in a single cell, or from the cell organization into non-anastomic organs such as the liver. We propose to take advantage of the smallness of the ratio of a cardiomyocyte length to the myocardium size in the one hand, and of the observed repetitive arrangement of cells in the other hand, to derive a global constitutive law for the myocardial tissue. Such a procedure belongs to the field of homogenization techniques whose general description can be found in Bensoussan et al. [2], Cioranescu and Saint Jean Paulin [10], Sanchez-Palencia [22]. It allows to replace a genuine discrete model of the myocardium equilibrium that would take into account all myocytes separately by a continuous medium model. The method relies on the knowledge of the mechanical behaviour at the microscopical level. In other words, we need to precise the mechanical modelling of both the cardiomyocytes and the interactions between cardiomyocytes. Stretching of the cardiac cells is mainly responsible or the elastic behaviour of the myocardium. But it cannot explain elastic effects that are transverse to the myocyte directions. In the present modelling, transverse effects are taken into account through interactions between connected myocytes. More precisely, we model myocytes by extensible bars that remain straight and interactions by moments between these bars. These moments are partly due to the bending of myocytes that we do not explicitly introduce in our modelling. Actually, bending effects seem to be weak and experimentally out of reach. From a purely formal point of view, bending can be added at the expense of a much more complicated macroscopical model, see for instance [21]. Let us mention that from an experimental point of view mechanical data on isolated cells become available, see, for instance, Zile et al. [30].

Homogenization methods were applied during the last decades to many physical settings, such as composite materials or trusses. Several techniques for proving convergence results were developed altogether. Here, we follow the discrete homogenization approach originated in Tollenaere and Caillerie [25], and in Moreau and Caillerie [17]. This approach is designed for discrete structures. Let us mention that Briane [4] was, up to our knowledge, the first to use homogenization techniques for a cardiac tissue modelling. In his work, the 


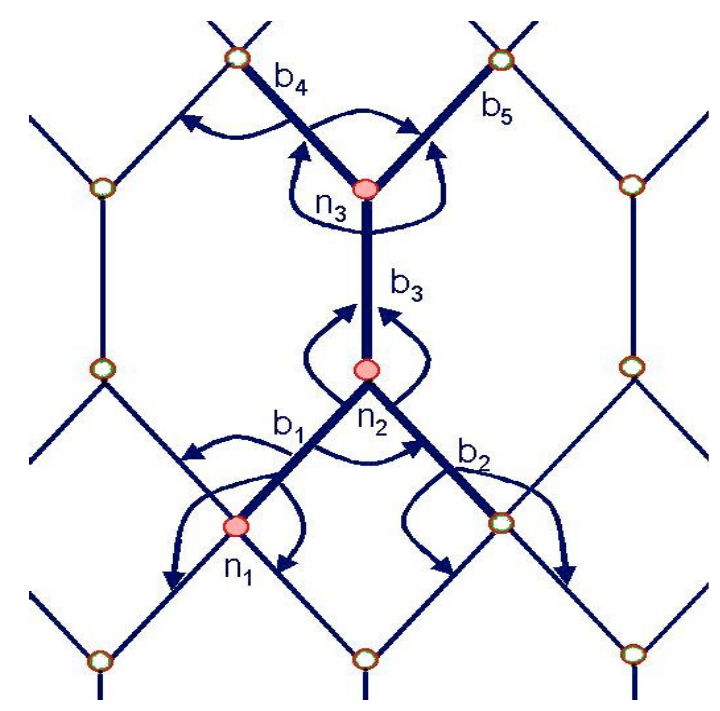

Figure 2. In bold, an elementary cell with three nodes colored in red and five bars: $\mathcal{N}_{\mathcal{R}}=\left\{n_{1}, n_{2}, n_{3}\right\}, \mathcal{B}_{\mathcal{R}}=\left\{b_{1}, b_{2}, b_{3}, b_{4}, b_{5}\right\}$. There are twelve interactions: $\mathcal{C}_{\mathcal{R}}=$ $\left\{c_{1}, c_{2}, c_{3}, c_{4}, c_{5}, c_{6}, c_{7}, c_{8}, c_{9}, c_{10}, c_{11}, c_{12}\right\}$.

microscopic level is the fibre level, and the framework is linearized elasticity. We restrict our analysis to a formal derivation.

\section{Myocyte ARRAngement And LATtice GeOMEtry}

\subsection{Numbering and connections of lattice elements}

We model a set of cardiomyocytes by a lattice of bars linked at their ends. The bar junctions are the lattice nodes. In order to write the lattice equilibrium system, we first need to identify the elements of the mechanical system - here, the lattice nodes and the bars - as well as their interactions. We introduce in the following a way of numbering nodes, bars and pairs of interacting bars that will be convenient in the use of the discrete homogenization method. It will allow to reflect the repetitive display of cardiomyocytes. These numberings are the discrete analogs of reference configurations in continuum mechanics.

From now on we use the word "cell" with its classical meaning in the context of homogenization theory. No confusion should arise with cardiac biological cells that we preferably call cardiomyocytes. Let us first describe an infinite lattice. We consider lattices whose reference configurations (sets of numbers in our modelling) are obtained by the repetition over $\mathbb{Z}^{3}$ of a given elementary cell (a finite set of numbers in our modelling), that we call the reference cell. See a bidimensional example in Figure 2. This cell contains "nodes" and "bars". Their respective numbers range over finite subsets $\mathcal{N}_{\mathcal{R}}$ and $\mathcal{B}_{\mathcal{R}}$ of $\mathbb{N}$. With any $\nu=\left(\nu^{1}, \nu^{2}, \nu^{3}\right) \in \mathbb{Z}^{3}$, we associate the $\nu$-cell which also comprises Card $\mathcal{N}_{\mathcal{R}}$ nodes and Card $\mathcal{B}_{\mathcal{R}}$ bars. Therefore, nodes and bars of the whole of the infinite lattice are now numbered by quadruples $\tilde{n}=\left(n, \nu^{1}, \nu^{2}, \nu^{3}\right)$ in $\mathcal{N}_{\mathcal{R}} \times \mathbb{Z}^{3}, \tilde{b}=\left(b, \nu^{1}, \nu^{2}, \nu^{3}\right)$ in $\mathcal{B}_{\mathcal{R}} \times \mathbb{Z}^{3}$. This means that the node (resp. bar) referred to by $\tilde{n}$ (resp. $\tilde{b}$ ) is the node (resp. bar) with number $n$ (resp. $b$ ) in the $\nu$-cell. We let $\tilde{\mathcal{N}}^{\infty}=\mathcal{N}_{\mathcal{R}} \times \mathbb{Z}^{3}$ and $\tilde{\mathcal{B}}^{\infty}=\mathcal{B}_{\mathcal{R}} \times \mathbb{Z}^{3}$, and, in a slightly improper way, we say that $\tilde{\mathcal{N}}^{\infty}$ (resp. $\tilde{\mathcal{B}}^{\infty}$ ) is the set of nodes (resp. bars) of the infinite lattice.

Let us now specify in which way nodes in $\tilde{\mathcal{N}}^{\infty}$ connect to each other. First of all, we assume that each bar in $\tilde{\mathcal{B}}^{\infty}$ links two nodes and with each bar $\tilde{b}$ we associate an origin node $O(\tilde{b})$ and an end node $E(\tilde{b})$. From our above description, any node $O(\tilde{b})$ and any node $E(\tilde{b})$ can necessarily be written under the form of a quadruple. We assume that the origin node $O(\tilde{b})$ of a bar $\tilde{b}=\left(b, \nu^{1}, \nu^{2}, \nu^{3}\right)$ associated with the cell $\left(\nu^{1}, \nu^{2}, \nu^{3}\right)$ belongs 
TABLE 1. Bar numbering.

\begin{tabular}{c|ccccc}
\hline$b$ & $b_{1}$ & $b_{2}$ & $b_{3}$ & $b_{4}$ & $b_{5}$ \\
\hline$O_{\mathrm{R}}(b)$ & $n_{1}$ & $n_{2}$ & $n_{2}$ & $n_{3}$ & $n_{3}$ \\
$E_{\mathrm{R}}(b)$ & $n_{2}$ & $n_{1}$ & $n_{3}$ & $n_{1}$ & $n_{1}$ \\
$\delta(b)$ & $(0,0)$ & $(1,0)$ & $(0,0)$ & $(0,1)$ & $(1,1)$ \\
\hline
\end{tabular}

to this very cell. Therefore, there exists $n$ such that $O\left(\left(b, \nu^{1}, \nu^{2}, \nu^{3}\right)\right)=\left(n, \nu^{1}, \nu^{2}, \nu^{3}\right)$. Moreover, in order to express the repetitivity of the lattice, we impose that the integer $n$ depends only on $b$. It coincides with the number of the origin node of the bar $b$ in the reference cell and can be denoted by $O_{\mathrm{R}}(b)$. On the contrary, the end node $E(\tilde{b})$ that we can write under the form $\left(m, \mu^{1}, \mu^{2}, \mu^{3}\right)$ does not necessarily belong to the same cell as $\tilde{b}$. In all cases, it belongs to a cell that can be numbered by $\left(\nu^{1}+\delta^{1}, \nu^{2}+\delta^{2}, \nu^{3}+\delta^{3}\right)$, where $\left(\delta^{1}, \delta^{2}, \delta^{3}\right) \in \mathbb{Z}^{3}$. Again, as the lattice is repetitive, $m, \delta^{1}, \delta^{2}$ and $\delta^{3}$ depend only on $b$ and they are denoted by $E_{\mathrm{R}}(b), \delta^{1 b}, \delta^{2 b}$ and $\delta^{3 b}$. In other words,

$$
O\left(\left(b, \nu^{1}, \nu^{2}, \nu^{3}\right)\right)=\left(O_{\mathrm{R}}(b), \nu^{1}, \nu^{2}, \nu^{3}\right), \quad E\left(\left(b, \nu^{1}, \nu^{2}, \nu^{3}\right)\right)=\left(E_{\mathrm{R}}(b), \nu^{1}+\delta^{1 b}, \nu^{2}+\delta^{2 b}, \nu^{3}+\delta^{3 b}\right) .
$$

An example of such a numbering for the bidimensional example of Figure 2 is given in Table 1.

Notice that we could have described the lattice in the following equivalent way. Consider two finite subsets $\mathcal{N}_{\mathcal{R}}$ and $\mathcal{B}_{\mathcal{R}}$ of $\mathbb{N}$, respectively called the set of (numbers of) reference nodes and the set of (numbers of) reference bars. Choose two mappings $O_{\mathrm{R}}, E_{\mathrm{R}}: \mathcal{B}_{\mathcal{R}} \mapsto \mathcal{N}_{\mathcal{R}}$ and a mapping $\delta: \mathcal{B}_{\mathcal{R}} \mapsto \mathbb{Z}^{3}$ such that for any $b \in \mathcal{B}_{\mathcal{R}}$, $\left(O_{\mathrm{R}}(b), 0\right) \neq\left(E_{\mathrm{R}}(b), \delta(b)\right)$ and such that the mapping $O_{\mathrm{R}} \times\left(E_{\mathrm{R}}, \delta\right)$ is one-to-one. Then, the set of (numbers of) nodes (resp. bars) of the associated infinite lattice is defined by $\tilde{\mathcal{N}}^{\infty}=\mathcal{N}_{\mathcal{R}} \times \mathbb{Z}^{3}=\left\{\tilde{n}=(n, \nu) ; n \in \mathcal{N}_{\mathcal{R}}, \nu \in \mathbb{Z}^{3}\right\}$ (resp. $\tilde{\mathcal{B}}^{\infty}=\mathcal{B}_{\mathcal{R}} \times \mathbb{Z}^{3}=\left\{\tilde{b}=(b, \nu) ; b \in \mathcal{B}_{\mathcal{R}}, \nu \in \mathbb{Z}^{3}\right\}$ ). Any $\nu \in \mathbb{Z}^{3}$ is said to define the $\nu$-cell whose set of nodes (resp. bars) is given by $\left\{(n, \nu) ; n \in \mathcal{N}_{\mathcal{R}}\right\}$ (resp. $\left\{(b, \nu) ; b \in \mathcal{B}_{\mathcal{R}}\right\}$ ). The overall origin and end mappings $O, E: \tilde{\mathcal{B}}^{\infty} \mapsto \tilde{\mathcal{N}}^{\infty}$ are defined by

$$
\forall \tilde{b}=(b, \nu) \in \tilde{\mathcal{B}}^{\infty}, O((b, \nu))=\left(O_{\mathrm{R}}(b), \nu\right), E((b, \nu))=\left(\left(E_{\mathrm{R}}(b), \nu+\delta(b)\right) .\right.
$$

When a bar belongs to a cell numbered by $\nu$, its origin belongs to this very cell as well.

With the above numberings for nodes and bars, a reference configuration of an infinite lattice is well defined. For future use, let us introduce a way of numbering interactions between bars. Such a numbering can necessarily be written in terms of the previous definitions, but this would lead to cumbersome notation. When writing mechanical balance in an actual configuration, we will assume in the sequel that any two bars sharing a common end mechanically interact. From the repetitivity of the reference configuration, any given bar $(b, \nu)$ in $\tilde{\mathcal{B}}^{\infty}$ interacts with a finite number of bars, and this number does not depend on $\nu$. It depends on $b$ only. Moreover, if a bar $(b, \nu)$ interacts with $\left(b^{\prime}, \nu^{\prime}\right)$, then for any $\mu$ in $\mathbb{Z}^{3},(b, \mu)$ interacts with $\left(b^{\prime}, \mu+\nu^{\prime}-\nu\right)$. It follows that the overall set of interactions between connected bars can be numbered by a set

$$
\tilde{\mathcal{C}}^{\infty}=\left\{(c, \nu) ; c \in \mathcal{C}_{\mathcal{R}}, \nu \in \mathbb{Z}^{3}\right\},
$$

where any $c \in \mathcal{C}_{\mathcal{R}} \subset \mathbb{N}$ refers to the interaction between bars that can be written $(b(c), \nu)$ and $\left(b^{\prime}(c), \nu+\gamma(c)\right)$. In such a numbering, care is devoted not to take into account an overall interaction twice. To this aim, it is convenient to consider that all interactions $\tilde{c}$ in the lattice occur between a first bar $P(\tilde{c})$ and a second bar $D(\tilde{c})$. A repeated interaction referred to by $c$ operates between a first bar $\left(P_{\mathrm{R}}(c), \nu\right)$ and a second bar $\left(D_{\mathrm{R}}(c), \nu+\gamma(c)\right)$. To sum up, all interactions $\tilde{c}=(c, \nu)$ read alternately $\tilde{c}=(P(\tilde{c}), D(\tilde{c}))$ with

$$
P(\tilde{c})=\left(P_{\mathrm{R}}(c), \nu^{1}, \nu^{2}, \nu^{3}\right), \quad D(\tilde{c})=\left(D_{\mathrm{R}}(c), \nu^{1}+\gamma^{1 c}, \nu^{2}+\gamma^{2 c}, \nu^{3}+\gamma^{3 c}\right) .
$$

An example of such a numbering is given in Table 2. Notice that our method easily extends to other cases. 
TABLE 2. Bar interaction numbering.

\begin{tabular}{c|cccccccccccc}
\hline$c$ & $c_{1}$ & $c_{2}$ & $c_{3}$ & $c_{4}$ & $c_{5}$ & $c_{6}$ & $c_{7}$ & $c_{8}$ & $c_{9}$ & $c_{10}$ & $c_{11}$ & $c_{12}$ \\
\hline$P_{\mathrm{R}}(c)$ & $b_{1}$ & $b_{1}$ & $b_{2}$ & $b_{3}$ & $b_{3}$ & $b_{4}$ & $b_{1}$ & $b_{1}$ & $b_{1}$ & $b_{2}$ & $b_{2}$ & $b_{4}$ \\
$D_{\mathrm{R}}(c)$ & $b_{2}$ & $b_{3}$ & $b_{3}$ & $b_{4}$ & $b_{5}$ & $b_{5}$ & $b_{2}$ & $b_{4}$ & $b_{5}$ & $b_{4}$ & $b_{5}$ & $b_{5}$ \\
$\gamma(c)$ & $(0,0)$ & $(0,0)$ & $(0,0)$ & $(0,0)$ & $(0,0)$ & $(0,0)$ & $(-1,0)$ & $(0,-1)$ & $(-1,-1)$ & $(1,-1)$ & $(0,-1)$ & $(-1,0)$ \\
\hline
\end{tabular}

For instance, we could assume that some connections between bars are activated and some others are not. In so far that this pattern is repetitive, it can be easily included in the definition of the set $\mathcal{C}_{\mathcal{R}} \subset \mathbb{N}$ and of the mappings $P_{\mathrm{R}}, D_{\mathrm{R}}: \mathcal{C}_{\mathcal{R}} \mapsto \mathcal{B}_{\mathcal{R}}$ and $\gamma: \mathcal{C}_{\mathcal{R}} \mapsto \mathbb{Z}^{3}$.

We now turn to finite lattices that we will actually consider in the remaining part of this work. Following the general homogenization technique, we introduce a sequence of reference configurations parametrized by $\varepsilon$. Let $\omega$ be a domain in $\mathbb{R}^{3}$. For any $\varepsilon$, we define a subset $Z^{\varepsilon}$ of $\mathbb{Z}^{3}$ by

$$
Z^{\varepsilon}=\left\{\nu=\left(\nu^{1}, \nu^{2}, \nu^{3}\right) \in \mathbb{Z}^{3} ; \varepsilon \nu \in \omega\right\}
$$

This defines the cells of a finite net. We define a reference configuration associated with $\varepsilon$ by its set of nodes $\tilde{\mathcal{N}}^{\varepsilon}=\mathcal{N}_{\mathcal{R}} \times Z^{\varepsilon}$ and by its set of bars $\tilde{\mathcal{B}}^{\varepsilon}=\left\{\tilde{b}=(b, \nu) \in \mathcal{B}_{\mathcal{R}} \times Z^{\varepsilon} ; E(\tilde{b}) \in \tilde{\mathcal{N}}^{\varepsilon}\right\}$. The overall interactions are then described by $\tilde{\mathcal{C}}^{\varepsilon}=\left\{\tilde{c}=(c, \nu) \in \mathcal{C}_{\mathcal{R}} \times Z^{\varepsilon} ; D(\tilde{c}) \in \tilde{\mathcal{B}}^{\varepsilon}\right\}$. Notice that $\nu$-cells "close to the boundary" of $\omega$ are not the exact repetition of the reference cell. Some bars and some interactions are ignored on purpose. This is of no consequence for the homogenization process which deals with inner cells and disregards boundary conditions.

In the sequel, we will use the notation $\lambda^{\varepsilon}=\left(\varepsilon \nu^{1}, \varepsilon \nu^{2}, \varepsilon \nu^{3}\right)$. The cells of an $\varepsilon$-lattice are, according to Truesdell terminology [26], labelled either by $\nu \in Z^{\varepsilon}$ or by $\lambda^{\varepsilon} \in \omega$. Therefore, $\nu$ or $\lambda^{\varepsilon}$ play the role of discrete Lagrangian variables. The Lagrangian configuration of the continuous medium to be defined will be $\omega$ and its Lagrangian variable will be $\lambda=\left(\lambda^{1}, \lambda^{2}, \lambda^{3}\right)$.

\subsection{Actual geometry description}

In the previous subsection, we defined the mechanical elements of the lattices and we identified them with numbers. The geometry of a lattice in a given state is then fully defined once positions of nodes are given. We choose an origin in the physical space identified with $\mathbb{R}^{3}$ and we denote the position of any node $\tilde{n} \in \tilde{\mathcal{N}}^{\varepsilon}$ by $\mathbf{R}^{\varepsilon}(\tilde{n})$. For any bar $\tilde{b} \in \tilde{\mathcal{B}}^{\varepsilon}$, we define a branch vector $\mathbf{B}^{\varepsilon \tilde{b}}$, a length $l^{\varepsilon \tilde{b}}$ and a unit vector $\mathbf{e}^{\varepsilon \tilde{b}}$ by

$$
\mathbf{B}^{\varepsilon \tilde{b}}=\mathbf{R}^{\varepsilon}(E(\tilde{b}))-\mathbf{R}^{\varepsilon}(O(\tilde{b})), \quad l^{\varepsilon \tilde{b}}=\left\|\mathbf{B}^{\varepsilon \tilde{b}}\right\|, \quad \mathbf{e}^{\varepsilon \tilde{b}}=\frac{\mathbf{B}^{\varepsilon \tilde{b}}}{l^{\varepsilon \tilde{b}}} .
$$

\section{Mechanical modelling}

\subsection{Internal and external forces}

The lattice is a mechanical system which consists of nodes and bars. Let us describe the internal efforts it can undergo. We momentarily drop out the $\varepsilon$ exponent.

Nodes are points. Therefore, they can be submitted to forces only. We assume that they interact with the bars they are linked to. Consider a bar $\tilde{b}$ with an end $\tilde{n}$. We denote by $\mathbf{f}^{\tilde{b} / \tilde{n}}$ the force exerted by $\tilde{b}$ onto $\tilde{n}$. Then, by virtue of the action-reaction principle, the force $\mathbf{f}^{\tilde{n} / \tilde{b}}=-\mathbf{f}^{\tilde{b} / \tilde{n}}$ is exerted by the node $\tilde{n}$ onto the bar $\tilde{b}$. As for pairs of bars $\left(\tilde{b}, \tilde{b^{\prime}}\right)$ sharing a common end, we assume that they interact by means of moments. Let $\mathbf{M}^{\tilde{b} / \tilde{b}^{\prime}}$ be the moment exerted by $\tilde{b}$ on $\tilde{b^{\prime}}$ at their common end, then the action-reaction principle states that $\mathbf{M}^{\tilde{b^{\prime}} / \tilde{b}}=-\mathbf{M}^{\tilde{b} / \tilde{b^{\prime}}}$.

We assume that the only external efforts acting on the lattice are forces $\mathbf{f}^{e / \tilde{n}}$ exerted on nodes. 


\subsection{Balance equations}

We restrict our analysis to a static framework, which means that we neglect the inertia terms. The lattice equilibrium comes down to the equilibrium of each element of the mechanical system. Let us first consider the balance of nodes. For any $\tilde{n}$, it reads

$$
\sum_{\tilde{b} \in O^{-1}(\tilde{n}) \cup E^{-1}(\tilde{n})} \mathbf{f}^{\tilde{b} / \tilde{n}}+\mathbf{f}^{e / \tilde{n}}=0
$$

The equilibrium of any bar $\tilde{b}$ results from the balance of forces and from the balance of moments it is submitted to. The balance of forces reads

$$
\mathbf{f}^{O(\tilde{b}) / \tilde{b}}+\mathbf{f}^{E(\tilde{b}) / \tilde{b}}=0 .
$$

Setting, for each $\tilde{b}, \mathbf{T}^{\tilde{b}}=\mathbf{f}^{E(\tilde{b}) / \tilde{b}}$, we immediately see that $\mathbf{f}^{O(\tilde{b}) / \tilde{b}}=-\mathbf{T}^{\tilde{b}}$. Inserting this relation in $(3)$ and using the action-reaction principle for forces, equations $((3)-(4))$ simplify in

$$
\forall \tilde{n} \in \tilde{\mathcal{N}}, \sum_{\tilde{b} \in O^{-1}(\tilde{n})} \mathbf{T}^{\tilde{b}}-\sum_{\tilde{b} \in E^{-1}(\tilde{n})} \mathbf{T}^{\tilde{b}}+\mathbf{f}^{e / \tilde{n}}=0 .
$$

The balance of moments exerted by all bars interacting with $\tilde{b}$ can be expressed at any point in $\mathbb{R}^{3}$. We choose to express it at $O(\tilde{b})$. Setting, for each $\tilde{c}, \mathbf{M}^{\tilde{c}}=\mathbf{M}^{D(\tilde{c}) / P(\tilde{c})}$, and using the action-reaction principle for moments, it reads

$$
\forall \tilde{b} \in \tilde{\mathcal{B}}, \sum_{\tilde{c} \in P^{-1}(\tilde{b})} \mathbf{M}^{\tilde{c}}-\sum_{\tilde{c} \in D^{-1}(\tilde{b})} \mathbf{M}^{\tilde{c}}+l^{\tilde{b}} \mathbf{e}^{\tilde{b}} \wedge \mathbf{T}^{\tilde{b}}=0 .
$$

Equations ((5)-(6)) are the strong formulation of the balance equations. For an easy use of the homogenization technique, they are preferably written in their weak formulation, also called virtual power formulation. This weak formulation is classically obtained by multiplying (5) by virtual translations $\mathbf{v}(\tilde{n}) \in \mathbb{R}^{3}$, by multiplying (6) by virtual rotation velocities $\mathbf{w}(\tilde{b}) \in \mathbb{R}^{3}$ and by summing over $\tilde{n}$ and $\tilde{b}$. A change of summation which can be seen as a discrete integration by parts yields the following system

$$
\begin{array}{r}
\forall \mathbf{v}(\cdot): \tilde{\mathcal{N}} \mapsto \mathbb{R}^{3}, \quad \sum_{\tilde{b} \in \tilde{\mathcal{B}}} \mathbf{T}^{\tilde{b}} \cdot[\mathbf{v}(O(\tilde{b}))-\mathbf{v}(E(\tilde{b}))]+\sum_{\tilde{n} \in \tilde{\mathcal{N}}} \mathbf{f}^{e / \tilde{n}} \cdot \mathbf{v}(\tilde{n})=0, \\
\forall \mathbf{w}(\cdot): \tilde{\mathcal{B}} \mapsto \mathbb{R}^{3}, \quad \sum_{\tilde{c} \in \tilde{\mathcal{C}}} \mathbf{M}^{\tilde{c}} \cdot[\mathbf{w}(P(\tilde{c}))-\mathbf{w}(D(\tilde{c}))]+\sum_{\tilde{b} \in \tilde{\mathcal{B}}} l^{\tilde{b}}\left(\mathbf{e}^{\tilde{b}} \wedge \mathbf{T}^{\tilde{b}}\right) \cdot \mathbf{w}(\tilde{b})=0,
\end{array}
$$

where $\cdot$ is the inner product in $\mathbb{R}^{3}$.

\subsection{Constitutive equations}

We can uniquely decompose the effort $\mathbf{T}^{\tilde{b}}$ into its axial (or normal) and transversal components with respect to $\mathbf{e}^{\tilde{b}}$ and write

$$
\mathbf{T}^{\tilde{b}}=N^{\tilde{b}} \mathbf{e}^{\tilde{b}}+\mathbf{T}_{t}^{\tilde{b}}, \quad \text { with } \quad \mathbf{T}_{t}^{\tilde{b}} \cdot \mathbf{e}^{\tilde{b}}=0
$$

We assume that the axial force $N^{\tilde{b}}$ in any bar $\tilde{b}$ depends on the actual positions of both its ends. The principle of frame invariance then implies that $N^{\tilde{b}}$ is a function of their distance $l^{\tilde{b}}$ only. We express the repetitivity of the lattice by assuming that a single such constitutive function $\mathcal{N}^{b}$ is valid for all bars $\tilde{b}$ with a same $b \in \mathcal{B}_{\mathcal{R}}$. More precisely, we assume that

$$
N^{\tilde{b}}=\mathcal{N}^{b}\left(l^{\tilde{b}}, l_{0}^{\tilde{b}}\right),
$$

where we make the constitutive equation for $N^{\tilde{b}}$ depend explicitly on a parameter $l_{0}^{\tilde{b}}$ that allows to model the cardiomyocytes contraction. This parameter is the length at rest of $\tilde{b}$ defined by $\mathcal{N}^{b}\left(l_{0}^{\tilde{b}}, l_{0}^{\tilde{b}}\right)=0$. A simple and 
mechanically meaningful choice consists in assuming that there exists a function $\overline{\mathcal{N}}^{b}$ defined on $\mathbb{R}^{+}$satisfying $\overline{\mathcal{N}}^{b}(1)=0$ and such that

or, equivalently, that

$$
\forall\left(l, l_{0}\right) \in \mathbb{R}^{+2}, \mathcal{N}^{b}\left(l, l_{0}\right)=\overline{\mathcal{N}}^{b}\left(\frac{l}{l_{0}}\right)
$$

$$
\forall\left(l, l_{0}\right) \in \mathbb{R}^{+2}, \forall k \in \mathbb{R}^{+}, \mathcal{N}^{b}\left(k l, k l_{0}\right)=\mathcal{N}^{b}\left(l, l_{0}\right) .
$$

Similarly, we assume that moments $\mathbf{M}^{\tilde{c}}$ between interacting bars depend on the unit vectors $\mathbf{e}^{P(\tilde{c})}$ and $\mathbf{e}^{D(\tilde{c})}$. From the principle of frame invariance again, and from the repetitivity hypothesis, we obtain that the constitutive equations read

$$
\mathbf{M}^{\tilde{c}}=\mathcal{M}^{c}\left(p^{\tilde{c}}\right) \mathbf{e}^{P(\tilde{c})} \wedge \mathbf{e}^{D(\tilde{c})}, \text { where } p^{\tilde{c}}=\mathbf{e}^{P(\tilde{c})} \cdot \mathbf{e}^{D(\tilde{c})} .
$$

As will be seen in the sequel, the shearing component $\mathbf{T}_{t}^{\tilde{b}}$ can be eliminated from the set of equations ((7)-(8)). It is a Lagrange multiplier of this formulation. It is therefore natural that it is not involved in any constitutive relation.

\subsection{Deformation problem}

When submitted to external forces and to some boundary conditions, the lattice deforms. The deformation problem consists of balance equations ((7)-(8)), of constitutive equations ((10) and (13)), and of possible conditions on some node positions. The primary unknowns are the node positions $\mathbf{R}(\tilde{n})$.

It has to be stressed out that the shearing forces $\mathbf{T}_{t}^{\tilde{b}}$ are unknowns as well. As usual in the analysis of a problem with Lagrange multipliers (see Brezzi and Fortin [3], for instance) they can be eliminated by taking in ((7)-(8)) kinematically admissible virtual velocities. In the present lattice problem, the admissibility condition comes from the fact that, by equation (2), the branch vectors $\mathbf{B}^{\tilde{b}}$ are completely determined from the node positions $\mathbf{R}(\tilde{n})$ and, that the rotation velocities of bars are, in turn, determined from the node velocities. Therefore, the kinematical admissibility condition for virtual velocities is expected to be

$$
\mathbf{w}(\tilde{b})=\frac{1}{\left(l^{\tilde{b}}\right)^{2}}\left[\mathbf{B}^{\tilde{b}} \wedge(\mathbf{v}(E(\tilde{b}))-\mathbf{v}(O(\tilde{b})))\right] .
$$

That such a choice for the test-functions actually allows to eliminate $\mathbf{T}_{t}^{\tilde{b}}$ can readily be seen by adding (7) and (8) restricted to pairs $(\mathbf{v}(\cdot), \mathbf{w}(\cdot))$ satisfying the compatibility condition (14). Terms containing $\mathbf{T}_{t}^{\tilde{b}}$ cancel out.

Once the shearing forces eliminated, the only unknowns are the node positions. Assuming that the resulting problem is well-posed and is solved, then it remains to go back to the equilibrium system and to solve in $\mathbf{T}_{t}^{\tilde{b}}$. The vectorial multiplication of equation (2.6) by $\mathbf{e}^{\tilde{b}}$, for instance, gives

$$
\forall \tilde{b} \in \tilde{\mathcal{B}}, \quad \mathbf{T}_{t}^{\tilde{b}}=\frac{1}{l^{\tilde{b}}}\left[\sum_{\tilde{c} \in D^{-1}(\tilde{b})} \mathbf{M}^{\tilde{c}}-\sum_{\tilde{c} \in P^{-1}(\tilde{b})} \mathbf{M}^{\tilde{c}}\right] \wedge \mathbf{e}^{\tilde{b}} .
$$

\section{Asymptotic expansions}

\subsection{Asymptotic expansions of node positions}

As explained in the introduction, we intend to take advantage of the scale separation to model cardiomyocyte lattices by a continuous medium. We already introduced the homogenization procedure which consists in considering a sequence of lattices parametrized by $\varepsilon$ and in identifying the unknowns, and, more importantly, the model that their leading terms satisfy by means of asymptotic expansions. 
We recall that $\omega$ is a domain in $\mathbb{R}^{3}$, and that, for any $\varepsilon>0, Z^{\varepsilon}$ and $\tilde{\mathcal{N}}^{\varepsilon}$ are defined by $Z^{\varepsilon}=\left\{\nu=\left(\nu^{1}, \nu^{2}, \nu^{3}\right) \in\right.$ $\left.\mathbb{Z}^{3} ; \varepsilon \nu \in \omega\right\}$ and $\tilde{\mathcal{N}}^{\varepsilon}=\mathcal{N}_{\mathcal{R}} \times Z^{\varepsilon}$. When considered for an $\varepsilon$-lattice, the equilibrium equations or constitutive equations that we gave in $((7)-(8)$ and $(10))$ and (13) will be called $\left(\left(7_{\varepsilon}\right)-\left(8_{\varepsilon}\right)\right.$ and $\left.\left(10_{\varepsilon}\right)\right)$ and $\left(13_{\varepsilon}\right)$. We assume that in any deformed state the lattices stay quasiperiodic. Then, the discrete homogenization method relies on the Ansatz that, for any $n \in \mathcal{N}_{\mathcal{R}}$, there exist vector functions $\mathbf{R}^{0}, \mathbf{R}^{n 1}, \mathbf{R}^{n 2}, \ldots$ defined on $\omega$ such that, for any $\varepsilon>0$ and for any $\tilde{n} \in \tilde{\mathcal{N}}^{\varepsilon}$, the actual node positions can be expanded as

$$
\mathbf{R}^{\varepsilon}(\tilde{n})=\mathbf{R}^{0}\left(\lambda^{\varepsilon}\right)+\varepsilon \mathbf{R}^{n 1}\left(\lambda^{\varepsilon}\right)+\varepsilon^{2} \mathbf{R}^{n 2}\left(\lambda^{\varepsilon}\right)+\cdots,
$$

where $\tilde{n}=(n, \nu)$ and $\lambda^{\varepsilon}=\varepsilon \nu$. Notice that we assume that $\mathbf{R}^{0}$ does not depend on $n$. This means that this leading term is the same for all nodes in the cell numbered by $\nu$. Therefore, it locates the actual position of this cell, and consequently will be interpreted as the deformation function of the equivalent continuous medium. The following terms $\mathbf{R}^{n 1}, \mathbf{R}^{n 2}, \ldots$ of the expansions depend on $n$, they give at different orders the position of the node $n$ of the cell $\nu$ relatively to $\mathbf{R}^{0}\left(\lambda^{\varepsilon}\right)$.

Expansion (16) easily induces expansions for the branch vectors $\mathbf{B}^{\varepsilon \tilde{b}}=\mathbf{R}^{\varepsilon}(E(\tilde{b}))-\mathbf{R}^{\varepsilon}(O(\tilde{b}))$. Indeed, from (16), we have simultaneously

$$
\mathbf{R}^{\varepsilon}(O(\tilde{b}))=\mathbf{R}^{0}\left(\lambda^{\varepsilon}\right)+\varepsilon \mathbf{R}^{O_{\mathrm{R}}(b) 1}\left(\lambda^{\varepsilon}\right)+\varepsilon^{2} \mathbf{R}^{O_{\mathrm{R}}(b) 2}\left(\lambda^{\varepsilon}\right)+\cdots,
$$

and

$$
\mathbf{R}^{\varepsilon}(E(\tilde{b}))=\mathbf{R}^{0}\left(\lambda^{\varepsilon}+\varepsilon \delta^{b}\right)+\varepsilon \mathbf{R}^{E_{\mathrm{R}}(b) 1}\left(\lambda^{\varepsilon}+\varepsilon \delta^{b}\right)+\varepsilon^{2} \mathbf{R}^{E_{\mathrm{R}}(b) 2}\left(\lambda^{\varepsilon}+\varepsilon \delta^{b}\right)+\cdots
$$

Adding some regularity assumptions on $\mathbf{R}^{0}$, we can write that

$$
\mathbf{R}^{0}\left(\lambda^{\varepsilon}+\varepsilon \delta^{b}\right)=\mathbf{R}^{0}\left(\lambda^{\varepsilon}\right)+\varepsilon \frac{\partial \mathbf{R}^{0}\left(\lambda^{\varepsilon}\right)}{\partial \lambda^{j}} \delta^{j b}+\cdots
$$

It follows that

$$
\forall \varepsilon, \forall \tilde{b} \in \tilde{\mathcal{B}}^{\varepsilon}, \mathbf{B}^{\varepsilon \tilde{b}}=\varepsilon \mathbf{B}^{b 0}\left(\lambda^{\varepsilon}\right)+\varepsilon^{2} \mathbf{B}^{b 1}\left(\lambda^{\varepsilon}\right)+\cdots,
$$

where $\mathbf{B}^{b 0}: \omega \mapsto \mathbb{R}^{3}$ is defined by

$$
\forall \lambda \in \omega, \mathbf{B}^{b 0}(\lambda)=\mathbf{R}^{E_{\mathrm{R}}(b) 1}(\lambda)-\mathbf{R}^{O_{\mathrm{R}}(b) 1}(\lambda)+\frac{\partial \mathbf{R}^{0}(\lambda)}{\partial \lambda^{j}} \delta^{j b} .
$$

This immediately yields asymptotic expansions for $l^{\varepsilon \tilde{b}}$ and $\mathbf{e}^{\varepsilon \tilde{b}}$. Namely,

$$
\forall \varepsilon, \forall \tilde{b} \in \tilde{\mathcal{B}}^{\varepsilon}, l^{\varepsilon \tilde{b}}=\varepsilon l^{b 0}\left(\lambda^{\varepsilon}\right)+\varepsilon^{2} l^{b 1}\left(\lambda^{\varepsilon}\right)+\cdots, \text { and }, \mathbf{e}^{\varepsilon \tilde{b}}=\mathbf{e}^{b 0}\left(\lambda^{\varepsilon}\right)+\varepsilon \mathbf{e}^{b 1}\left(\lambda^{\varepsilon}\right)+\cdots,
$$

where $l^{b 0}: \omega \mapsto \mathbb{R}$ and $\mathbf{e}^{b 0}: \omega \mapsto \mathbb{R}^{3}$ are defined by $l^{b 0}=\left\|\mathbf{B}^{b 0}\right\|$, and $\mathbf{e}^{b 0}=\frac{\mathbf{B}^{b 0}}{l^{b 0}}$.

In order to be consistent with (22), we assume moreover that, for any $b \in \mathcal{B}_{\mathcal{R}}$, there exists $l_{0}^{b}: \omega \mapsto \mathbb{R}^{+}$such that the length at rest $l_{0}^{\tilde{b}}$ of any $\tilde{b} \in \tilde{\mathcal{B}}^{\varepsilon}$ can be written as

$$
l_{0}^{\tilde{b}}=\varepsilon l_{0}^{b}\left(\lambda^{\varepsilon}\right) .
$$

\subsection{Asymptotic expansions of tensions and moments}

The expansions of $l^{\varepsilon \tilde{b}}$ and $\mathbf{e}^{\varepsilon \tilde{b}}$ that we obtained in the previous section provide expansions for the efforts by means of the constitutive equations $\left(10_{\varepsilon}\right)$ and $\left(13_{\varepsilon}\right)$. Nevertheless, we first need to state how the constitutive laws $\mathcal{N}^{\varepsilon b}$ and $\mathcal{M}^{\varepsilon c}$ depend on $\varepsilon$. In the same way the dependence on $\varepsilon$ of the external forces $\mathbf{f}^{\varepsilon e / \tilde{n}}$ has to be precised. 
We assume that, for any $b \in \mathcal{B}_{\mathcal{R}}$, the constitutive law $\mathcal{N}^{\varepsilon b}$ is, as a mapping from $\mathbb{R}^{2}$ into $\mathbb{R}$, of order 0 in $\varepsilon$. Recall that the second variable, which is present to take into account the length at rest, is in fact a parameter. More precisely, we assume that there exists $\mathcal{N}^{b 0}: \mathbb{R}^{2} \mapsto \mathbb{R}$ such that

$$
\forall \varepsilon, \forall b \in \mathcal{B}_{\mathcal{R}}, \forall\left(l, l_{0}\right) \in \mathbb{R}^{+2}, \mathcal{N}^{\varepsilon b}\left(l, l_{0}\right)=\mathcal{N}^{b 0}\left(l, l_{0}\right),
$$

where, following (12),

$$
\forall\left(l, l_{0}\right) \in \mathbb{R}^{+2}, \forall k \in \mathbb{R}^{+}, \mathcal{N}^{b 0}\left(k l, k l_{0}\right)=\mathcal{N}^{b 0}\left(l, l_{0}\right) .
$$

This is an arbitrary choice with no consequence on our analysis. What is important and can lead to several continuous models is the relative weight of the orders of magnitude in $\varepsilon$ of $\mathcal{N}^{\varepsilon b}$ in the one hand, and of $\mathcal{M}^{\varepsilon c}$ in the other hand. If the moments stiffness is too weak with respect to the tensions stiffness, then the moments do not appear in the equivalent continuous model, and, if in addition the lattice is not triangulated, the equivalent continuous model is singular due to the internal mechanisms. On the contrary, if the moments stiffness is too strong, then the continuous model does not take into account the bar tensions. The choice that gives the "richest" continuous model incorporating both bar tensions and moments consists in choosing an order of magnitude of 1 for the moments. In other words, we assume that there exists $\mathcal{M}^{c 0}:[-1,1] \mapsto \mathbb{R}$ such that

$$
\forall \varepsilon, \forall c \in \mathcal{C}_{\mathcal{R}}, \forall p \in[-1,1], \quad \mathcal{M}^{\varepsilon c}(p)=\varepsilon \mathcal{M}^{c 0}(p) .
$$

Finally, we take $\mathbf{f}^{\varepsilon e / \tilde{n}}$ of order 1 and such that

$$
\forall \varepsilon, \forall \tilde{n} \in \tilde{\mathcal{N}}^{\varepsilon}, \quad \mathbf{f}^{\varepsilon e / \tilde{n}}=\varepsilon \mathbf{f}^{e / n}\left(\lambda^{\varepsilon}\right) .
$$

Let us examine the consequences of choices (24) and (26), and of the asymptotic expansions obtained in Section 4.1, on the orders of magnitude of tensions and moments. From (22) and (24), and making use of property (25), we obtain that

$$
\forall \varepsilon, \forall \tilde{b} \in \tilde{\mathcal{B}}^{\varepsilon}, N^{\varepsilon \tilde{b}} \mathbf{e}^{\varepsilon \tilde{b}}=\mathcal{N}^{b 0}\left(l^{b 0}\left(\lambda^{\varepsilon}\right), l_{0}^{b}\left(\lambda^{\varepsilon}\right)\right) \mathbf{e}^{b 0}\left(\lambda^{\varepsilon}\right)+\varepsilon[\cdots]+\cdots
$$

Using (22) and (26), we obtain that

$$
\forall \varepsilon, \forall \tilde{c} \in \tilde{\mathcal{C}}^{\varepsilon}, \mathbf{M}^{\varepsilon \tilde{c}}=\varepsilon \mathcal{M}^{c 0}\left(p^{c 0}\left(\lambda^{\varepsilon}\right)\right) \mathbf{e}^{P_{\mathrm{R}}(c) 0}\left(\lambda^{\varepsilon}\right) \wedge \mathbf{e}^{D_{\mathrm{R}}(c) 0}\left(\lambda^{\varepsilon}\right)+\varepsilon^{2}[\cdots]+\cdots,
$$

where $p^{c 0}=\mathbf{e}^{P_{\mathrm{R}}(c) 0} \cdot \mathbf{e}^{D_{\mathrm{R}}(c) 0}: \omega \mapsto \mathbb{R}$. We recall that, as explained previously in Sections 3.3 and 3.4 , the shearing forces can be eliminated and can be obtained by solving the balance equations. This shows that (up to their free component) they have the same order of magnitude in $\varepsilon$ as the tensions $N^{\varepsilon \tilde{b}} \mathbf{e}^{\varepsilon \tilde{b}}$. We conveniently assign the same order to the possible free component. Then, we can write

$$
\forall \varepsilon, \forall \tilde{b} \in \tilde{\mathcal{B}}^{\varepsilon}, \mathbf{T}_{t}^{\varepsilon \tilde{b}}=\mathbf{T}_{t}^{b 0}\left(\lambda^{\varepsilon}\right)+\varepsilon[\cdots]+\cdots
$$

Finally, letting

$$
\mathbf{T}^{b 0}=\mathcal{N}^{b 0}\left(l^{b 0}, l_{0}^{b}\right) \mathbf{e}^{b 0}+\mathbf{T}_{t}^{b 0}: \omega \mapsto \mathbb{R}^{3}, \text { and, } \mathbf{M}^{c 0}=\mathcal{M}^{c 0}\left(p^{c 0}\right) \mathbf{e}^{P_{\mathrm{R}}(c) 0} \wedge \mathbf{e}^{D_{\mathrm{R}}(c) 0}: \omega \mapsto \mathbb{R}^{3},
$$

we obtain the expansions we aimed at

$$
\forall \varepsilon, \forall \tilde{b} \in \tilde{\mathcal{B}}^{\varepsilon}, \forall \tilde{c} \in \tilde{\mathcal{C}}^{\varepsilon}, \mathbf{T}^{\varepsilon \tilde{b}}=\mathbf{T}^{b 0}\left(\lambda^{\varepsilon}\right)+\varepsilon[\cdots]+\cdots, \mathbf{M}^{\varepsilon \tilde{c}}=\varepsilon \mathbf{M}^{c 0}\left(\lambda^{\varepsilon}\right)+\varepsilon^{2}[\cdots]+\cdots
$$




\section{Cauchy Stress tensor and Balance EQUations OF THE EQUIVALENT CONTINUOUS MEDIUM}

The internal efforts in the lattices are tensions and moments. In a continuous medium, all efforts are described by a stress tensor field, that is a mapping defined on a reference configuration (or on the deformed configuration) with values in $\left(\mathbb{R}^{3}\right)^{3}$ (or in the set $\mathbb{M}_{3}$ of real $3 \times 3$ matrices). This section is devoted to define a stress tensor from the discrete sets of bar tensions and moments. In the same time, we derive the partial differential balance equations fulfilled by the stresses. First, we obtain the internal efforts of the continuous medium and its balance equations in the curvilinear Lagrangian representation given by the variable $\left(\lambda^{1}, \lambda^{2}, \lambda^{3}\right)$. Then, we write our results in the usual space representation.

\subsection{Curvilinear Lagrangian representation}

Balance equations. The balance equations of the continuous medium are obtained by letting $\varepsilon$ go to 0 in the virtual power formulation $\left(\left(7_{\varepsilon}\right)-\left(8_{\varepsilon}\right)\right)$ of the lattices equilibrium. They will yield the definition of the internal continuum efforts.

To this aim, we choose in $\left(7_{\varepsilon}\right)$ virtual velocities $\mathbf{v}^{\varepsilon}(\tilde{n})$ that coincide with the values at $\lambda^{\varepsilon}$ of a smooth virtual macroscopic velocity field and we let $\varepsilon$ go to 0 . Notice that a technical difficulty arises. Indeed, equations $\left(7_{\varepsilon}\right)$ involve sums $\sum_{\tilde{n} \in \tilde{\mathcal{N}}^{\varepsilon}}\left(\operatorname{resp} . \sum_{\tilde{b} \in \tilde{\mathcal{B}}^{\varepsilon}}\right.$ ) over the sets of all nodes (resp. bars) of the lattices, whose cardinals tend to infinity when $\varepsilon$ goes to zero. To settle this question, we split such sums in two successive sums $\sum_{\nu^{i} \in Z^{\varepsilon}} \sum_{n \in \mathcal{N}_{\mathcal{R}}}$ (resp. $\sum_{\nu^{i} \in Z^{\varepsilon}} \sum_{b \in \mathcal{B}_{\mathcal{R}}}$ ) over all cells and over all nodes (resp. bars) of each cell. Upon multiplication by $\varepsilon^{3}$, any sum $\sum_{\nu^{i} \in Z^{\varepsilon}}$ over all cells may be interpreted as a Riemann sum of an integral over $\omega$. Therefore, for any $g$ smooth enough, $\varepsilon^{3} \sum_{\nu^{i} \in Z^{\varepsilon}} g\left(\nu^{i} \varepsilon\right)$ goes to $\int_{\omega} g(\lambda) \mathrm{d} \lambda$, when $\varepsilon$ goes to 0 .

More precisely, let $\mathbf{v}: \omega \mapsto \mathbb{R}^{3}$ be a macroscopic virtual velocity field equal to zero on $\partial \omega$, and, for any $\varepsilon$, for any $\tilde{n} \in \tilde{\mathcal{N}}^{\varepsilon}$, let $\mathbf{v}^{\varepsilon}(\tilde{n})=\mathbf{v}\left(\lambda^{\varepsilon}\right)$. A Taylor expansion yields:

$$
\mathbf{v}^{\varepsilon}(O(\tilde{b}))-\mathbf{v}^{\varepsilon}(E(\tilde{b}))=\mathbf{v}\left(\lambda^{\varepsilon}\right)-\mathbf{v}\left(\lambda^{\varepsilon}+\varepsilon \delta^{i b}\right)=-\varepsilon \frac{\partial \mathbf{v}}{\partial \lambda^{i}}\left(\lambda^{\varepsilon}\right) \delta^{i b}+\cdots
$$

Inserting in $\left(7_{\varepsilon}\right)$, making $\varepsilon$ go to 0 , and letting $\mathbf{f}=\sum_{n \in \mathcal{N}_{\mathcal{R}}} \mathbf{f}^{e / n}$, we obtain the virtual power formulation of the balance equation of the equivalent continuous medium. It reads:

$$
\forall \mathbf{v}: \omega \mapsto \mathbb{R}^{3}, \mathbf{v}_{\mid \partial \omega}=0, \quad-\int_{\omega} \mathbf{S}^{i 0} \cdot \frac{\partial \mathbf{v}}{\partial \lambda^{i}} \mathrm{~d} \lambda+\int_{\omega} \mathbf{f} \cdot \mathbf{v} \mathrm{d} \lambda=0,
$$

where, for any $i=1,2,3$, we define $\mathbf{S}^{i 0}: \omega \mapsto \mathbb{R}^{3}$ by

$$
\mathbf{S}^{i 0}=\sum_{b \in \mathcal{B}_{\mathcal{R}}} \mathbf{T}^{b 0} \delta^{i b}
$$

The three vectors $\mathbf{S}^{i 0}$ have to be interpreted as the stress vectors describing the internal efforts of a continuous medium in a parametric Lagrangian representation, see Washizu [28]. Equation (35) gives their definition in terms of the internal forces of a discrete structure. For more detail about this definition, see [6].

A first symmetry property. We deal with $\left(8_{\varepsilon}\right)$ in the same way. Let $\mathbf{w}: \omega \mapsto \mathbb{R}^{3}$ be a macroscopic rotation velocity field and, for any $\varepsilon$, for any $\tilde{b}=(b, \nu) \in \tilde{\mathcal{B}}^{\varepsilon}$, let $\mathbf{w}^{\varepsilon}(\tilde{b})=\mathbf{w}\left(\lambda^{\varepsilon}\right)$. Then, the difference $\mathbf{w}^{\varepsilon}(P(\tilde{c}))-\mathbf{w}^{\varepsilon}(D(\tilde{c}))$ is of order 1 and, from $(32), \mathbf{M}^{\varepsilon \tilde{c}} \cdot\left[\mathbf{w}^{\varepsilon}(P(\tilde{c}))-\mathbf{w}^{\varepsilon}(D(\tilde{c}))\right]$ is of order 2 . Therefore, the expansion of $\left(8_{\varepsilon}\right)$ yields, in the set of mappings from $\omega$ into $\mathbb{R}^{3}$, the identity

$$
\sum_{b \in \mathcal{B}_{\mathcal{R}}} \mathbf{B}^{b 0} \wedge \mathbf{T}^{b 0}=0
$$


Denoting by $L^{A}$ the skew-symmetric part of an endomorphism $L$, equation (36) is equivalent to

$$
\sum_{b \in \mathcal{B}_{\mathcal{R}}}\left(\mathbf{B}^{b 0} \otimes \mathbf{T}^{b 0}\right)^{A}=0
$$

Indeed, for any $\mathbf{a}$ and $\mathbf{b}$ in $\mathbb{R}^{3}, \mathbf{a} \otimes \mathbf{b}$ is defined by: $(\mathbf{a} \otimes \mathbf{b}) \mathbf{c}=(\mathbf{b} \cdot \mathbf{c}) \mathbf{a}$ for any $\mathbf{c}$ in $\mathbb{R}^{3}$. Therefore,

$$
(\mathbf{a} \wedge \mathbf{b}) \wedge \mathbf{c}=(\mathbf{a} \cdot \mathbf{c}) \mathbf{b}-(\mathbf{b} \cdot \mathbf{c}) \mathbf{a}=(\mathbf{b} \otimes \mathbf{a}) \mathbf{c}-(\mathbf{a} \otimes \mathbf{b}) \mathbf{c}=2(\mathbf{b} \otimes \mathbf{a})^{A} \mathbf{c}, \text { and },[\mathbf{a} \wedge \mathbf{b}=0] \Leftrightarrow\left[(\mathbf{b} \otimes \mathbf{a})^{A}=0\right]
$$

Result (37) will be used in the proof of the symmetry of the Cauchy stress tensor, see Section 5.3.

Another expression of the stress vectors. The stress vectors $\mathbf{S}^{i 0}, i=1,2,3$, have been defined by equation (35), where any $\mathbf{T}^{b 0}, b \in \mathcal{B}_{\mathcal{R}}$, is given by equation (31). This equation contains terms $\mathbf{T}_{t}^{b 0}$ which cannot follow a constitutive law, and that will actually be unknowns on the lattice problem on the reference cell, see Section 6.2. Let us write out another expression of $\mathbf{S}^{i 0}, i=1,2,3$, that does not involve these terms.

Let $\mathbf{v}^{i}: \omega \mapsto \mathbb{R}^{3}, i=1,2,3$, be three macroscopic vector fields and now choose in $\left(8_{\varepsilon}\right)$ virtual rotation velocities $\mathbf{w}^{\varepsilon}(\tilde{b})$ such that, for any $\varepsilon$, for any $\tilde{b}=\left(b, \nu^{1}, \nu^{2}, \nu^{3}\right)$,

$$
\mathbf{w}^{\varepsilon}(\tilde{b})=\varepsilon \frac{1}{l^{b}} \mathbf{e}^{\tilde{b}} \wedge \mathbf{v}^{i}(\varepsilon \nu) \delta^{i b}
$$

We know from $(22)$ that $\mathbf{w}^{\varepsilon}(\tilde{b})$ expands as

$$
\mathbf{w}^{\varepsilon}(\tilde{b})=\left(\frac{1}{l^{b 0}} \mathbf{e}^{b 0} \wedge \mathbf{v}^{i} \delta^{i b}\right)\left(\lambda^{\varepsilon}\right)+\cdots
$$

Therefore, the two terms of $\left(8_{\varepsilon}\right)$ expand as

$$
\begin{aligned}
\mathbf{M}^{\varepsilon \tilde{c}} \cdot\left[\mathbf{w}^{\varepsilon}(P(\tilde{c}))-\mathbf{w}^{\varepsilon}(D(\tilde{c}))\right] & =\varepsilon \mathbf{M}^{c 0} \cdot\left[\left(\frac{\mathbf{e}^{P_{\mathrm{R}}(c) 0}}{l^{P_{\mathrm{R}}(c) 0}} \delta^{i P_{\mathrm{R}}(c)}-\frac{\mathbf{e}^{D_{\mathrm{R}}(c) 0}}{l^{D_{\mathrm{R}}(c) 0}} \delta^{i D_{\mathrm{R}}(c)}\right) \wedge \mathbf{v}^{i}\right]+\cdots \\
& =\varepsilon\left[\mathbf{M}^{c 0} \wedge\left(\frac{\mathbf{e}^{P_{\mathrm{R}}(c) 0}}{l^{P_{\mathrm{R}}(c) 0}} \delta^{i P_{\mathrm{R}}(c)}-\frac{\mathbf{e}^{D_{\mathrm{R}}(c) 0}}{l^{D_{\mathrm{R}}(c) 0}} \delta^{i D_{\mathrm{R}}(c)}\right)\right] \cdot \mathbf{v}^{i}+\cdots
\end{aligned}
$$

and

$$
\begin{aligned}
l^{\tilde{b}}\left(\mathbf{e}^{\tilde{b}} \wedge \mathbf{T}^{\tilde{b}}\right) \cdot \mathbf{w}^{\varepsilon}(\tilde{b}) & =\varepsilon\left(\mathbf{e}^{b 0} \wedge \mathbf{T}^{b 0}\right) \cdot\left(\mathbf{e}^{b 0} \wedge \mathbf{v}^{i} \delta^{i b}\right)+\cdots=\varepsilon\left[\left(\mathbf{e}^{b 0} \wedge \mathbf{T}^{b 0}\right) \wedge \mathbf{e}^{b 0}\right] \cdot \mathbf{v}^{i} \delta^{i b}+\cdots \\
& =\varepsilon\left[\mathbf{T}^{b 0}-\left(\mathbf{T}^{b 0} \cdot \mathbf{e}^{b 0}\right) \mathbf{e}^{b 0}\right] \cdot \mathbf{v}^{i} \delta^{i b}+\cdots=\varepsilon \mathbf{T}_{t}^{b 0} \cdot \mathbf{v}^{i} \delta^{i b}+\cdots
\end{aligned}
$$

Summing over $\tilde{\mathcal{C}}^{\varepsilon}$ and $\tilde{\mathcal{B}}^{\varepsilon}$, and using the convergence of Riemann sums towards volume integrals, we obtain the expected relation between the leading terms of the moments and the leading terms of the shearing forces. Namely,

$$
\forall \mathbf{v}^{i}: \omega \mapsto \mathbb{R}^{3}, i=1,2,3, \quad \int_{\omega}\left[\sum_{c \in \mathcal{C}_{\mathcal{R}}} \mathbf{M}^{c 0} \wedge\left(\frac{\mathbf{e}^{P_{\mathrm{R}}(c) 0}}{l^{P_{\mathrm{R}}(c) 0}} \delta^{i P_{\mathrm{R}}(c)}-\frac{\mathbf{e}^{D_{\mathrm{R}}(c) 0}}{l^{D_{\mathrm{R}}(c) 0}} \delta^{i D_{\mathrm{R}}(c)}\right)+\sum_{b \in \mathcal{B}_{\mathcal{R}}} \mathbf{T}_{t}^{b 0} \delta^{i b}\right] \cdot \mathbf{v}^{i} \mathrm{~d} \lambda=0
$$

or equivalently,

$$
\sum_{b \in \mathcal{B}_{\mathcal{R}}} \mathbf{T}_{t}^{b 0} \delta^{i b}=-\sum_{c \in \mathcal{C}_{\mathcal{R}}} \mathbf{M}^{c 0} \wedge\left[\frac{\mathbf{e}^{P_{\mathrm{R}}(c) 0}}{l^{P_{\mathrm{R}}(c) 0}} \delta^{i P_{\mathrm{R}}(c)}-\frac{\mathbf{e}^{D_{\mathrm{R}}(c) 0}}{l^{D_{\mathrm{R}}(c) 0}} \delta^{i D_{\mathrm{R}}(c)}\right]
$$


Definition (35) of $\mathbf{S}^{i 0}$, which can be written as $\mathbf{S}^{i 0}=\sum_{b \in \mathcal{B}_{\mathcal{R}}}\left(N^{b 0} \mathbf{e}^{b 0}+\mathbf{T}_{t}^{b 0}\right) \delta^{i b}$, now becomes

$$
\mathbf{S}^{i 0}=\sum_{b \in \mathcal{B}_{\mathcal{R}}} N^{b 0} \mathbf{e}^{b 0} \delta^{i b}+\sum_{c \in \mathcal{C}_{\mathcal{R}}} \mathbf{M}^{c 0} \wedge\left[\frac{\mathbf{e}^{D_{\mathrm{R}}(c) 0}}{l^{D_{\mathrm{R}}(c) 0}} \delta^{i D_{\mathrm{R}}(c)}-\frac{\mathbf{e}^{P_{\mathrm{R}}(c) 0}}{l^{P_{\mathrm{R}}(c) 0}} \delta^{i P_{\mathrm{R}}(c)}\right] .
$$

\subsection{Space representation and Cauchy stress tensor}

In the homogenization process, the discrete variables $\left(\lambda^{1 \varepsilon}, \lambda^{2 \varepsilon}, \lambda^{3 \varepsilon}\right)=\left(\varepsilon \nu^{1}, \varepsilon \nu^{2}, \varepsilon \nu^{3}\right), \nu \in Z^{\varepsilon}$, become continuous variables $\left(\lambda^{1}, \lambda^{2}, \lambda^{3}\right)$ which label the material points of the equivalent continuous medium. From (16), we can see that the position in the physical space of a material point labeled by $\left(\lambda^{1}, \lambda^{2}, \lambda^{3}\right)$ is $\mathbf{R}^{0}\left(\lambda^{1}, \lambda^{2}, \lambda^{3}\right)$, and that the deformed space configuration of the continuous medium is $\Omega=\mathbf{R}^{0}(\omega)$. The primary description of the internal efforts of a continuous medium is given by a Cauchy stress tensor which is defined at each point of the deformed configuration and, at each point, is a linear operator of the physical space. Let us explain how the Cauchy stress tensor $\sigma^{0}: \Omega \mapsto L\left(\mathbb{R}^{3}\right)$ of the equivalent continuous medium can be expressed in terms of the vectors $\mathbf{S}^{i 0}: \omega \mapsto \mathbb{R}^{3}, i=1,2,3$.

It suffices to use $\mathbf{R}^{0}$ as a change of variables between the parametric Lagrangian configuration $\omega$ and the deformed space configuration $\Omega=\mathbf{R}^{0}(\omega)$. Let $g(\lambda)=\left(\frac{\partial \mathbf{R}^{0}}{\partial \lambda^{1}}, \frac{\partial \mathbf{R}^{0}}{\partial \lambda^{2}}, \frac{\partial \mathbf{R}^{0}}{\partial \lambda^{3}}\right)(\lambda)$ be the mixed product of the partial derivatives of $\mathbf{R}^{0}$. The usual space coordinates are denoted by $x$. With any $\mathbf{v}: \omega \mapsto \mathbb{R}^{3}$, we can associate $\mathbf{v} \circ\left(\mathbf{R}^{0}\right)^{-1}: \Omega \mapsto \mathbb{R}^{3}$. We loosely keep the same notation for both fields $\mathbf{v}$ and $\mathbf{v} \circ\left(\mathbf{R}^{0}\right)^{-1}$, and we denote by $\nabla_{x} \mathbf{v}$ the gradient tensor of $\mathbf{v}$ with respect to $x$. Obviously,

$$
\frac{\partial \mathbf{v}}{\partial \lambda^{i}}=\nabla_{x} \mathbf{v} \frac{\partial \mathbf{R}^{0}}{\partial \lambda^{i}}
$$

and the virtual power formulation (34) becomes:

$$
\forall \mathbf{v}: \Omega \mapsto \mathbb{R}^{3}, \quad-\int_{\Omega}\left(\mathbf{S}^{i 0} \otimes \frac{\partial \mathbf{R}^{0}}{\partial \lambda^{i}}\right): \nabla_{x} \mathbf{v} \frac{1}{g} \mathrm{~d} x+\int_{\Omega} \mathbf{f} \cdot \mathbf{v} \frac{1}{g} \mathrm{~d} x=0,
$$

where : denotes the scalar product of two tensors. Therefore, the Cauchy stress tensor $\boldsymbol{\sigma}^{0}$ is given by

$$
\boldsymbol{\sigma}^{0}=\frac{1}{g} \mathbf{S}^{i 0} \otimes \frac{\partial \mathbf{R}^{0}}{\partial \lambda^{i}}
$$

and satisfies the virtual power formulation of the continuous medium equilibrium

$$
\forall \mathbf{v}: \Omega \mapsto \mathbb{R}^{3}, \mathbf{v}_{\mid \partial \Omega}=0, \quad-\int_{\Omega} \boldsymbol{\sigma}^{0}: \nabla_{x} \mathbf{v}^{0} \mathrm{~d} x+\int_{\Omega} \frac{1}{g} \mathbf{f} \cdot \mathbf{v} \mathrm{d} x=0 .
$$

\subsection{Symmetry of the Cauchy stress tensor}

In continuum mechanics, the existence of a Cauchy stress tensor and the fact that it is symmetric are derived from first principles. Here, we recovered a Cauchy stress tensor from discrete tensions and moments. Let us check that it is actually symmetric.

Result. The Cauchy stress tensor $\boldsymbol{\sigma}^{0}$ coincides with $\frac{1}{g} \sum_{b \in \mathcal{B}_{\mathcal{R}}} \mathbf{T}^{b 0} \otimes \mathbf{B}^{b 0}$ and is symmetric.

Proof. Let $A(\cdot): \omega \mapsto \mathbb{M}_{3}$ be a smooth field of second order tensors and choose $\mathbf{v}(\tilde{n})=A\left(\lambda^{\varepsilon}\right) \mathbf{R}^{n 1}\left(\lambda^{\varepsilon}\right)$ in $\left(7_{\varepsilon}\right)$. The expansion of $\left(7_{\varepsilon}\right)$ leads to

$$
\int_{\omega}\left[\sum_{b \in \mathcal{B}_{\mathcal{R}}} \mathbf{T}^{b 0} \otimes\left(\mathbf{R}^{E_{\mathrm{R}}(b) 1}-\mathbf{R}^{O_{\mathrm{R}}(b) 1}\right)\right]: A \mathrm{~d} \lambda=0,
$$


or, equivalently,

$$
\sum_{b \in \mathcal{B}_{\mathcal{R}}} \mathbf{T}^{b 0} \otimes\left(\mathbf{R}^{E_{\mathrm{R}}(b) 1}-\mathbf{R}^{O_{\mathrm{R}}(b) 1}\right)=0 .
$$

From the definitions (47) and (35) of $\sigma^{0}$ and $\mathbf{S}^{i 0}$, and from (21), we have that

$$
\boldsymbol{\sigma}^{0}=\frac{1}{g} \mathbf{S}^{i 0} \otimes \frac{\partial \mathbf{R}^{0}}{\partial \lambda^{i}}=\frac{1}{g} \sum_{b \in \mathcal{B}_{\mathcal{R}}} \mathbf{T}^{b 0} \otimes\left(\mathbf{B}^{b 0}-\mathbf{R}^{E_{\mathrm{R}}(b) 1}+\mathbf{R}^{O_{\mathrm{R}}(b) 1}\right),
$$

so that (50) yields $\boldsymbol{\sigma}^{0}=\frac{1}{g} \sum_{b \in \mathcal{B}_{\mathcal{R}}} \mathbf{T}^{b 0} \otimes \mathbf{B}^{b 0}$, which by (37) is symmetric.

\section{Constitutive equations of the Equivalent Continuous medium - SELF-EQUILIBRIUM EQUATIONS}

To complete the equivalent continuum model of the lattice, we need to determine the stress-strain relation of the continuous medium. In the elastic setting we are dealing with, this means that we search for a constitutive relationship $\left(\lambda, \mathbf{F}=\left(\mathbf{F}^{1}, \mathbf{F}^{2}, \mathbf{F}^{3}\right)\right) \in \omega \times\left(\mathbb{R}^{3}\right)^{3} \mapsto \hat{\mathbf{S}}^{0}(\lambda, \mathbf{F}) \in\left(\mathbb{R}^{3}\right)^{3}$ such that, for any deformation $\mathbf{R}^{0}$ : $\omega \mapsto \mathbb{R}^{3}$, the stress vectors $\mathbf{S}^{i 0}, i=1,2,3$, are given, at any $\lambda \in \omega$, by $\mathbf{S}^{0}(\lambda)=\left(\mathbf{S}^{10}(\lambda), \mathbf{S}^{20}(\lambda), \mathbf{S}^{30}(\lambda)\right)=$ $\hat{\mathbf{S}}^{0}\left(\lambda, \frac{\partial \mathbf{R}^{0}}{\partial \lambda^{1}}(\lambda), \frac{\partial \mathbf{R}^{0}}{\partial \lambda^{2}}(\lambda), \frac{\partial \mathbf{R}^{0}}{\partial \lambda^{3}}(\lambda)\right)$. We actually gave in (35) and (44) expressions for $\mathbf{S}^{i 0}$. The reader remembers that terms in the right-hand side of these equations contain both $\frac{\partial \mathbf{R}^{0}}{\partial \lambda}$ and differences $\mathbf{R}^{E_{\mathrm{R}}(b) 1}-\mathbf{R}^{O_{\mathrm{R}}(b) 1}, b \in \mathcal{B}_{\mathcal{R}}$, see (21), (31). Therefore, we must go a step further and be able to express $\mathbf{R}^{E_{\mathrm{R}}(b) 1}-\mathbf{R}^{O_{\mathrm{R}}(b) 1}, b \in \mathcal{B}_{\mathcal{R}}$, in terms of $\frac{\partial \mathbf{R}^{0}}{\partial \lambda^{i}}$.

As usual in the homogenization of periodic media (see Sanchez [22], for instance), this is done through the solution of a mechanical problem "at the elementary cell level". This problem consists of the leading constitutive equations for bars and pairs of interacting bars that were obtained in Section 4.2, and of so called self-equilibrium equations of the reference cell. We begin by making out these balance equations.

\subsection{Self-equilibrium equations}

The reference cell self-equilibrium equations are derived from the lattice equilibrium equations, by means of convenient choices of the test-functions. Namely, for any $n \in \mathcal{N}_{\mathcal{R}}$, choose $\mathbf{v}^{n}$ in $\mathbb{R}^{3}$, and for any $b \in \mathcal{B}_{\mathcal{R}}$, choose $\mathbf{w}^{b}$ in $\mathbb{R}^{3}$, then, in $\left(\left(7_{\varepsilon}\right)-\left(8_{\varepsilon}\right)\right)$, take $\mathbf{v}^{\varepsilon}(\tilde{n})=\varepsilon \theta\left(\lambda^{\varepsilon}\right) \mathbf{v}^{n}$ and $\mathbf{w}^{\varepsilon}(\tilde{b})=\eta\left(\lambda^{\varepsilon}\right) \mathbf{w}^{b}$ where $\theta$ and $\eta$ are smooth scalar fields defined on $\omega$. Then,

$$
\forall \varepsilon, \forall \tilde{b} \in \tilde{\mathcal{B}}^{\varepsilon}, \mathbf{v}^{\varepsilon}(O(\tilde{b}))-\mathbf{v}^{\varepsilon}(E(\tilde{b}))=\varepsilon\left[\theta\left(\lambda^{\varepsilon}\right) \mathbf{v}^{O_{\mathrm{R}}(b)}-\theta\left(\lambda^{\varepsilon}+\varepsilon \delta^{b}\right) \mathbf{v}^{E_{\mathrm{R}}(b)}\right]
$$

and, by a Taylor expansion,

$$
\forall \varepsilon, \forall \tilde{b} \in \tilde{\mathcal{B}}^{\varepsilon}, \mathbf{v}^{\varepsilon}(O(\tilde{b}))-\mathbf{v}^{\varepsilon}(E(\tilde{b}))=\varepsilon \theta\left(\lambda^{\varepsilon}\right)\left[\mathbf{v}^{O_{\mathrm{R}}(b)}-\mathbf{v}^{E_{\mathrm{R}}(b)}\right]+\cdots
$$

In the same way, we have:

$$
\forall \varepsilon, \forall \tilde{c} \in \tilde{\mathcal{C}}^{\varepsilon}, \mathbf{w}^{\varepsilon}(P(\tilde{c}))-\mathbf{w}^{\varepsilon}(D(\tilde{c}))=\varepsilon \eta\left(\lambda^{\varepsilon}\right)\left[\mathbf{w}^{P_{\mathrm{R}}(c)}-\mathbf{w}^{D_{\mathrm{R}}(c)}\right]+\cdots
$$

Expanding $\left(\left(7_{\varepsilon}\right)-\left(8_{\varepsilon}\right)\right)$, we obtain by identification of the leading terms the following two equations:

$$
\forall \theta: \omega \mapsto \mathbb{R}, \forall \mathbf{v}^{n} \in \mathbb{R}^{3}, \quad \int_{\omega} \theta(\lambda) \sum_{b \in \mathcal{B}_{\mathcal{R}}} \mathbf{T}^{b 0}(\lambda) \cdot\left[\mathbf{v}^{O_{\mathrm{R}}(b)}-\mathbf{v}^{E_{\mathrm{R}}(b)}\right] \mathrm{d} \lambda=0,
$$




$$
\forall \eta: \omega \mapsto \mathbb{R}, \forall \mathbf{w}^{b} \in \mathbb{R}^{3}, \int_{\omega} \eta(\lambda)\left\{\sum_{c \in \mathcal{C}_{\mathcal{R}}} \mathbf{M}^{c 0}(\lambda) \cdot\left[\mathbf{w}^{P_{\mathrm{R}}(c)}-\mathbf{w}^{D_{\mathrm{R}}(c)}\right]+\sum_{b \in \mathcal{B}_{\mathcal{R}}}\left(\mathbf{B}^{b 0}(\lambda) \wedge \mathbf{T}^{b 0}(\lambda)\right) \cdot \mathbf{w}^{b}\right\} \mathrm{d} \lambda=0 .
$$

They are trivially equivalent to the following identities in the space of scalar functions defined on $\omega$ :

$$
\forall \mathbf{v}^{n} \in \mathbb{R}^{3}, n \in \mathcal{N}_{\mathcal{R}}, \quad \sum_{b \in \mathcal{B}_{\mathcal{R}}} \mathbf{T}^{b 0} \cdot\left[\mathbf{v}^{O_{\mathrm{R}}(b)}-\mathbf{v}^{E_{\mathrm{R}}(b)}\right]=0,
$$

and,

$$
\forall \mathbf{w}^{b} \in \mathbb{R}^{3}, b \in \mathcal{B}_{\mathcal{R}}, \quad \sum_{c \in \mathcal{C}_{\mathcal{R}}} \mathbf{M}^{c 0} \cdot\left[\mathbf{w}^{P_{\mathrm{R}}(c)}-\mathbf{w}^{D_{\mathrm{R}}(c)}\right]+\sum_{b \in \mathcal{B}_{\mathcal{R}}}\left(\mathbf{B}^{b 0} \wedge \mathbf{T}^{b 0}\right) \cdot \mathbf{w}^{b}=0 .
$$

Equations (57) and (58) are similar to the full lattice equilibrium equations ((7)-(8)) we started with. Instead of involving summations over the whole set of bars $\tilde{\mathcal{B}}^{\varepsilon}$ or over the whole set $\tilde{\mathcal{C}}^{\varepsilon}$, they only involve the sets $\mathcal{B}_{\mathcal{R}}$ and $\mathcal{C}_{\mathcal{R}}$ that are associated with the reference cell. Moreover, they do not contain any external loading, hence the denomination self-equilibrium equations.

\subsection{The reference cell problem. Derivation of the constitutive law}

The full reference cell problem consists of the above self-equilibrium equations and of the leading constitutive equations in the space of scalar or vector functions defined on $\omega$

$$
N^{b 0}=\mathcal{N}^{b 0}\left(l^{b 0}, l_{0}^{b}\right), \quad \mathbf{M}^{c 0}=\mathcal{M}^{c 0}\left(\mathbf{e}^{P_{\mathrm{R}}(c) 0} \cdot \mathbf{e}^{D_{\mathrm{R}}(c) 0}\right) \mathbf{e}^{P_{\mathrm{R}}(c) 0} \wedge \mathbf{e}^{D_{\mathrm{R}}(c) 0},
$$

where $N^{b 0}=\mathbf{T}^{b 0} \cdot \mathbf{e}^{b 0}$ and where, for any $b \in \mathcal{B}_{\mathcal{R}}, \mathbf{B}^{b 0}=\mathbf{R}^{E_{\mathrm{R}}(b) 1}-\mathbf{R}^{O_{\mathrm{R}}(b) 1}+\frac{\partial \mathbf{R}^{0}}{\partial \lambda^{j}} \delta^{j b}, l^{b 0}=\left\|\mathbf{B}^{b 0}\right\|$ and $\mathbf{e}^{b 0}=\frac{\mathbf{B}^{b 0}}{l^{b 0}}$. In the self-equilibrium equations $((57)-(58))$, the space variable obviously acts as a parameter. Indeed, for any $\lambda$ in $\omega$, the vectors $\mathbf{T}^{b 0}(\lambda), \mathbf{M}^{c 0}(\lambda)$ and $\mathbf{B}^{b 0}(\lambda)$ satisfy equations ((57)-(58)) taken at $\lambda$. A similar remark applies when equation (59) is added. For a given $\lambda$ and for a given value of $\frac{\partial \mathbf{R}^{0}}{\partial \lambda^{i}}(\lambda), i=1,2,3$, the whole set of equations determine, upon well-posedness, vector values that we can call $\mathbf{B}^{b 0}(\lambda), \mathbf{T}^{b 0}(\lambda), b \in \mathcal{B}_{\mathcal{R}}, \mathbf{M}^{c 0}(\lambda), c \in \mathcal{C}_{\mathcal{R}}$, which in turn determine $\left(\mathbf{S}^{10}(\lambda), \mathbf{S}^{20}(\lambda), \mathbf{S}^{30}(\lambda)\right)$. As $\frac{\partial \mathbf{R}^{0}}{\partial \lambda^{2}}(\lambda), i=1,2,3$, can be any $\left(\mathbf{F}^{1}, \mathbf{F}^{2}, \mathbf{F}^{3}\right)$ in $\left(\mathbb{R}^{3}\right)^{3}$, we have in fact built a mapping from $\omega \times\left(\mathbb{R}^{3}\right)^{3}$ into $\left(\mathbb{R}^{3}\right)^{3}$. This mapping is nothing but the constitutive law.

Let us, for convenience, and because this is the result we mainly aimed at, sum up the construction of this constitutive law. It reads: Let $\lambda$ be in $\omega$, and let $\mathbf{F}=\left(\mathbf{F}^{1}, \mathbf{F}^{2}, \mathbf{F}^{3}\right)$ be in $\left(\mathbb{R}^{3}\right)^{3}$. Find $\mathbf{R}^{n 1} \in \mathbb{R}^{3}, n \in \mathcal{N}_{\mathcal{R}}$, $\mathbf{T}^{b 0} \in \mathbb{R}^{3}, b \in \mathcal{B}_{\mathcal{R}}, \mathbf{M}^{c 0} \in \mathbb{R}^{3}, c \in \mathcal{C}_{\mathcal{R}}$, such that, letting, for any $b \in \mathcal{B}_{\mathcal{R}}, \mathbf{B}^{b 0}=\mathbf{R}^{E_{\mathrm{R}}(b) 1}-\mathbf{R}^{O_{\mathbb{R}}(b) 1}+\mathbf{F}^{j} \delta^{j b}$, $l^{b 0}=\left\|\mathbf{B}^{b 0}\right\|$ and $\mathbf{e}^{b 0}=\frac{\mathbf{B}^{b 0}}{l^{b 0}}$, the following equations in $\mathbb{R}$ or $\mathbb{R}^{3}$ are satisfied:

$$
\begin{aligned}
& \forall \mathbf{v}^{n} \in \mathbb{R}^{3}, \quad \sum_{b \in \mathcal{B}_{\mathcal{R}}} \mathbf{T}^{b 0} \cdot\left[\mathbf{v}^{O_{\mathrm{R}}(b)}-\mathbf{v}^{E_{\mathrm{R}}(b)}\right]=0, \\
& \forall \mathbf{w}^{b} \in \mathbb{R}^{3}, \quad \sum_{c \in \mathcal{C}_{\mathcal{R}}} \mathbf{M}^{c 0} \cdot\left[\mathbf{w}^{P_{\mathrm{R}}(c)}-\mathbf{w}^{D_{\mathrm{R}}(c)}\right]+\sum_{b \in \mathcal{B}_{\mathcal{R}}}\left(\mathbf{B}^{b 0} \wedge \mathbf{T}^{b 0}\right) \cdot \mathbf{w}^{b}=0, \\
& N^{b 0}=\mathcal{N}^{b 0}\left(l^{b 0}, l_{0}^{b}(\lambda)\right), \quad \mathbf{M}^{c 0}=\mathcal{M}^{c 0}\left(\mathbf{e}^{P_{\mathrm{R}}(c) 0} \cdot \mathbf{e}^{D_{\mathrm{R}}(c) 0}\right) \mathbf{e}^{P_{\mathrm{R}}(c) 0} \wedge \mathbf{e}^{D_{\mathrm{R}}(c) 0},
\end{aligned}
$$

where $N^{b 0}=\mathbf{T}^{b 0} \cdot \mathbf{e}^{b 0}$. Then, define

$$
\forall i=1,2,3, \mathbf{S}^{i 0}=\sum_{b \in \mathcal{B}_{\mathcal{R}}} N^{b 0} \mathbf{e}^{b 0} \delta^{i b}+\sum_{c \in \mathcal{C}_{\mathcal{R}}} \mathbf{M}^{c 0} \wedge\left[\frac{\mathbf{e}^{D_{\mathrm{R}}(c) 0}}{l^{D_{\mathrm{R}}(c) 0}} \delta^{i D_{\mathrm{R}}(c)}-\frac{\mathbf{e}^{P_{\mathrm{R}}(c) 0}}{l^{P_{\mathrm{R}}(c) 0}} \delta^{i P_{\mathrm{R}}(c)}\right] .
$$

As usual, the shearing vectors $\mathbf{T}_{t}^{b 0}$ can be eliminated and we are left with a problem whose unknowns are the vectors $\mathbf{R}^{n 1}, n \in \mathcal{N}_{\mathcal{R}}$. It is readily seen that a solution cannot be unique since these vectors appear only through 
their differences $\mathbf{R}^{E_{\mathrm{R}}(b) 1}-\mathbf{R}^{O_{\mathrm{R}}(b) 1}$. They can be, at best, uniquely determined up to an additive vector. Other singularities and loss of uniqueness or existence may appear, linked, for instance, to buckling or collapsing of the reference cell.

The set of the last four equations associates with any $\left(\lambda, \mathbf{F}=\left(\mathbf{F}^{1}, \mathbf{F}^{2}, \mathbf{F}^{3}\right)\right) \in \omega \times\left(\mathbb{R}^{3}\right)^{3}$ three vectors $\hat{\mathbf{S}}^{i 0}(\lambda, \mathbf{F})$ in $\mathbb{R}^{3}$. It defines the constitutive relationship of the continuous medium, which as expected is elastic. The variable $\lambda$ came into play through the length at rest and can make the constitutive law nonhomogeneous. Other nonhomogeneities could have been included in the myocyte laws.

It may be proved directly that, due to the frame invariance of the constitutive equations $((10)$ and $(13))$ for bars and for pairs of interacting bars, the equivalent medium constitutive equation is frame invariant as well. This feature can also be seen as a consequence of the frame invariance of the elastic potential determined in Section 7.

\subsection{Computation of the constitutive law}

Nonlinearity is present in two ways in problem $((60)-(62))$. First, the constitutive laws are nonlinear. Second, as we deal with large displacements, we did not linearize in our modelling terms such that $\mathbf{B}^{b 0} \wedge \mathbf{T}^{b 0}$, or $l^{b 0}$. This nonlinear problem can be solved, possibly after eliminating the shearing terms, with an iterative procedure. We implemented its resolution by Newton's method.

\section{Hyperelasticity}

We prove in this section that the equivalent continuous medium is hyperelastic, which means that its constitutive law derives from a potential, see, for instance, Ciarlet [9], Gurtin [12].

The constitutive equations for bars and for pairs of interacting bars have been defined in (10) and in (13) as mappings $l \mapsto \mathcal{N}^{b 0}\left(l, l_{0}^{b}\right)$ from $\mathbb{R}^{+}$into $\mathbb{R}$ and $p \mapsto \mathcal{M}^{c 0}(p)$ from $[-1,1]$ into $\mathbb{R}$. When performing asymptotic expansions, we already implicitly assumed that these mappings are continuous which is a mechanically sound hypothesis. Therefore, they are trivially the derivatives of some smooth functions. Let us define the potentials $W^{b}(\cdot)$ and $W^{c}(\cdot)$ by

$$
\forall l \in \mathbb{R}^{+}, \mathcal{N}^{b 0}(l)=\frac{\mathrm{d} W^{b}}{\mathrm{~d} l}(l), \quad \forall p \in[-1,1],-\mathcal{M}^{c 0}(p)=\frac{\mathrm{d} W^{c}}{\mathrm{~d} p}(p),
$$

where we omit, for convenience, the dependence in $l_{0}^{b}$. As mentioned in Section 6.2, the set of equations ((60)(62)) that defines the constitutive law associates (upon well-posedness) with any $\mathbf{F}=\left(\mathbf{F}^{1}, \mathbf{F}^{2}, \mathbf{F}^{3}\right) \in\left(\left(\mathbb{R}^{3}\right)^{3}\right.$ a set of vectors $\mathbf{B}^{b}(\mathbf{F})=\mathbf{R}^{E_{\mathrm{R}}(b) 1}-\mathbf{R}^{O_{\mathrm{R}}(b) 1}+\mathbf{F}^{j} \delta^{j b}$, where $b \in \mathcal{B}_{\mathcal{R}}$. With obvious notations, equations $((60)-(62))$ define for any $b \in \mathcal{B}_{\mathcal{R}}$ and for any $c \in \mathcal{C}_{\mathcal{R}}$ mappings

$$
\mathbf{F} \mapsto l^{b}(\mathbf{F}) \text { and } \mathbf{F} \mapsto p^{c}(\mathbf{F})=\mathbf{e}^{P_{\mathrm{R}}(c)}(\mathbf{F}) \cdot \mathbf{e}^{D_{\mathrm{R}}(c)}(\mathbf{F})
$$

It is well known that in a mechanical system energies are additive. This leads us to expect that an energy, if any, for the continuous medium is given by

$$
W\left(\mathbf{F}^{1}, \mathbf{F}^{2}, \mathbf{F}^{3}\right)=\sum_{b \in \mathcal{B}_{\mathcal{R}}} W^{b}\left(l^{b}\left(\mathbf{F}^{1}, \mathbf{F}^{2}, \mathbf{F}^{3}\right)\right)+\sum_{c \in \mathcal{C}_{\mathcal{R}}} W^{c}\left(p^{c}\left(\mathbf{F}^{1}, \mathbf{F}^{2}, \mathbf{F}^{3}\right)\right) .
$$

Result. The mapping $W=\sum_{b \in \mathcal{B}_{\mathcal{R}}} W^{b}\left(l^{b}(\cdot)\right)+\sum_{c \in \mathcal{C}_{\mathcal{R}}} W^{c}\left(p^{c}(\cdot)\right)$ defined on $\left(\mathbb{R}^{3}\right)^{3}$ and with values in $\mathbb{R}$ is an energy for the equivalent continuous model.

Proof. We have to prove that, for any $\mathbf{F}$ in $\left(\mathbb{R}^{3}\right)^{3}$, and for any $i=1,2,3, \hat{\mathbf{S}}^{i 0}(\mathbf{F})=\frac{\partial W}{\partial \mathbf{F}^{i}}(\mathbf{F})$ where $\hat{\mathbf{S}}^{i 0}$ is defined by equations ((60)-(63)). From definition (64), we see that we mainly have to differentiate $l^{b}$ and $p^{c}$ with respect 
to $\mathbf{F}^{i}, i=1,2,3$. We use incremental notations. For any $b \in \mathcal{B}_{R}$, let $\mathbf{U}^{b}=\mathbf{R}^{E_{\mathrm{R}}(b) 1}-\mathbf{R}^{O_{\mathrm{R}}(b) 1}$ and let us define $\mathrm{d} \mathbf{U}^{b}$, for any $b \in \mathcal{B}_{R}$, by

$$
\mathrm{d} \mathbf{U}^{b}=\mathbf{U}^{b}\left(\mathbf{F}^{1}+\mathrm{d} \mathbf{F}^{1}, \mathbf{F}^{2}+\mathrm{d} \mathbf{F}^{2}, \mathbf{F}^{3}+\mathrm{d} \mathbf{F}^{3}\right)-\mathbf{U}^{b}\left(\mathbf{F}^{1}, \mathbf{F}^{2}, \mathbf{F}^{3}\right) .
$$

Analogous definitions apply to $\mathrm{d} \mathbf{B}^{b}, \mathrm{~d} l^{b}, \mathrm{~d} \mathbf{e}^{b}, b \in \mathcal{B}_{R}$, and to $\mathrm{d} p^{c}, c \in \mathcal{C}_{R}$. Then, from the definitions of all mappings, we first obtain

$$
\mathrm{d} \mathbf{B}^{b}=\mathrm{d} \mathbf{U}^{b}+\mathrm{d} \mathbf{F}^{i} \delta^{i b}+\cdots, \quad \mathrm{d} l^{b}=\mathbf{e}^{b} \cdot \mathrm{d} \mathbf{B}^{b}+\cdots,
$$

which provides

$$
\mathrm{d} l^{b}=\mathbf{e}^{b} \cdot \mathrm{d} \mathbf{U}^{b}+\mathbf{e}^{b} \cdot \mathrm{d} \mathbf{F}^{i} \delta^{i b}+\cdots
$$

Then, we obtain

$$
\mathrm{d} \mathbf{e}^{b}=\frac{1}{l^{b}}\left[\mathrm{~d} \mathbf{B}^{b}-\left(\mathrm{d} \mathbf{B}^{b} \cdot \mathbf{e}^{b}\right) \mathbf{e}^{b}\right]+\cdots, \quad \mathrm{d} p^{c}=\mathbf{e}^{P_{\mathrm{R}}(c)} \cdot \mathrm{d} \mathbf{e}^{D_{\mathrm{R}}(c)}+\mathrm{d} \mathbf{e}^{P_{\mathrm{R}}(c)} \cdot \mathbf{e}^{D_{\mathrm{R}}(c)}+\cdots,
$$

from which we derive that

$$
\begin{aligned}
\mathrm{d} p^{c}= & {\left[\mathbf{e}^{P_{\mathrm{R}}(c)}-\left(\mathbf{e}^{P_{\mathrm{R}}(c)} \cdot \mathbf{e}^{D_{\mathrm{R}}(c)}\right) \mathbf{e}^{D_{\mathrm{R}}(c)}\right] \cdot \frac{\mathrm{d} \mathbf{B}^{D_{\mathrm{R}}(c)}}{l^{D_{\mathrm{R}}(c)}}+\left[\mathbf{e}^{D_{\mathrm{R}}(c)}-\left(\mathbf{e}^{D_{\mathrm{R}}(c)} \cdot \mathbf{e}^{P_{\mathrm{R}}(c)}\right) \mathbf{e}^{P_{\mathrm{R}}(c)}\right] \cdot \frac{\mathrm{d} \mathbf{B}^{P_{\mathrm{R}}(c)}}{l^{P_{\mathrm{R}}(c)}}+\cdots } \\
= & \left(\mathbf{e}^{P_{\mathrm{R}}(c)} \wedge \mathbf{e}^{D_{\mathrm{R}}(c)}\right) \cdot\left[\mathbf{e}^{P_{\mathrm{R}}(c)} \wedge \frac{\mathrm{d} \mathbf{B}^{P_{\mathrm{R}}(c)}}{l^{P_{\mathrm{R}}(c)}}-\mathbf{e}^{D_{\mathrm{R}}(c)} \wedge \frac{\mathrm{d} \mathbf{B}^{D_{\mathrm{R}}(c)}}{l^{D_{\mathrm{R}}(c)}}\right]+\cdots \\
= & \left(\mathbf{e}^{P_{\mathrm{R}}(c)} \wedge \mathbf{e}^{D_{\mathrm{R}}(c)}\right) \cdot\left[\left(\frac{\mathbf{e}^{P_{\mathrm{R}}(c)}}{l^{P_{\mathrm{R}}(c)}} \delta^{i P_{\mathrm{R}}(c)}-\frac{\mathbf{e}^{D_{\mathrm{R}}(c)}}{l^{D_{\mathrm{R}}(c)}} \delta^{i D_{\mathrm{R}}(c)}\right) \wedge \mathrm{d} \mathbf{F}^{i}\right. \\
& \left.+\frac{\mathbf{e}^{P_{\mathrm{R}}(c)}}{l^{P_{\mathrm{R}}(c)}} \wedge \mathrm{d} \mathbf{U}^{P_{\mathrm{R}}(c)}-\frac{\mathbf{e}^{D_{\mathrm{R}}(c)}}{l^{D_{\mathrm{R}}(c)}} \wedge \mathrm{d} \mathbf{U}^{D_{\mathrm{R}}(c)}\right]+\cdots
\end{aligned}
$$

where we used (65) in the last identity. Let us now differentiate $W$. By means of the chain rule, and using (66) and (68), we have

$$
\mathrm{d} W^{b}=\mathcal{N}^{b 0}\left(l^{b}, l_{0}^{b}\right)\left(e^{b} \cdot \mathrm{d} \mathbf{F}^{i} \delta^{i b}+e^{b} \cdot \mathrm{d} \mathbf{U}^{b}\right)
$$

and

$$
\begin{aligned}
\mathrm{d} W^{c}=-\mathcal{M}^{c 0}\left(p^{c}\right)\left(\mathbf{e}^{P_{\mathrm{R}}(c)} \wedge \mathbf{e}^{D_{\mathrm{R}}(c)}\right) \cdot\left[\left(\frac{\mathbf{e}^{P_{\mathrm{R}}(c)}}{l^{P_{\mathrm{R}}(c)}} \delta^{i P_{\mathrm{R}}(c)}-\right.\right. & \left.\frac{\mathbf{e}^{D_{\mathrm{R}}(c)}}{l^{D_{\mathrm{R}}(c)}} \delta^{i D_{\mathrm{R}}(c)}\right) \wedge \mathrm{d} \mathbf{F}^{i} \\
& \left.+\frac{\mathbf{e}^{P_{\mathrm{R}}(c)}}{l^{P_{\mathrm{R}}(c)}} \wedge \mathrm{d} \mathbf{U}^{P_{\mathrm{R}}(c)}-\frac{\mathbf{e}^{D_{\mathrm{R}}(c)}}{l^{D_{\mathrm{R}}(c)}} \wedge \mathrm{d} \mathbf{U}^{D_{\mathrm{R}}(c)}\right]+\cdots
\end{aligned}
$$

Letting $N^{b 0}=\mathcal{N}^{b 0}\left(l^{b}, l_{0}^{b}\right)$ and $\mathbf{M}^{c 0}=\mathcal{M}^{c 0}\left(p^{c}\right) \mathbf{e}^{P_{\mathrm{R}}(c)} \wedge \mathbf{e}^{D_{\mathrm{R}}(c)}$, we can conclude that

$$
\begin{aligned}
\mathrm{d} W= & \sum_{b \in \mathcal{B}_{\mathcal{R}}} \mathrm{d} W^{b}+\sum_{c \in \mathcal{C}_{\mathcal{R}}} \mathrm{d} W^{c} \\
= & {\left[\sum_{b \in \mathcal{B}_{\mathcal{R}}} N^{b 0} \mathbf{e}^{b} \delta^{i b}+\sum_{c \in \mathcal{C}_{\mathcal{R}}} \mathbf{M}^{c 0} \wedge\left(\frac{\mathbf{e}^{D_{\mathrm{R}}(c)}}{l^{D_{\mathrm{R}}(c)}} \delta^{i D_{\mathrm{R}}(c)}-\frac{\mathbf{e}^{P_{\mathrm{R}}(c)}}{l^{P_{\mathrm{R}}(c)}} \delta^{i P_{\mathrm{R}}(c)}\right)\right] \cdot \mathrm{d} \mathbf{F}^{i} } \\
& +\sum_{b \in \mathcal{B}_{\mathcal{R}}} N^{b 0} \mathbf{e}^{b} \cdot \mathrm{d} \mathbf{U}^{b}+\sum_{c \in \mathcal{C}_{\mathcal{R}}} \mathbf{M}^{c 0} \cdot\left[\frac{\mathbf{e}^{D_{\mathrm{R}}(c)}}{l^{D_{\mathrm{R}}(c)}} \wedge \mathrm{d} \mathbf{U}^{D_{\mathrm{R}}(c)}-\frac{\mathbf{e}^{P_{\mathrm{R}}(c)}}{l^{P_{\mathrm{R}}(c)}} \wedge \mathrm{d} \mathbf{U}^{P_{\mathrm{R}}(c)}\right]+\cdots
\end{aligned}
$$


From (44), the second line in (71) coincides with $\mathbf{S}^{i 0} \cdot \mathrm{d} \mathbf{F}^{i}$. The third one can be proved to be equal to 0 by choosing $\mathbf{v}^{n}=\mathrm{d} \mathbf{R}^{n 1}$ in the virtual power formulation of the reference cell equilibrium, when the shearing forces are eliminated. Therefore, we have actually proved that, for any $i=1,2,3, \mathbf{S}^{i 0}=\frac{\partial W}{\partial \mathbf{F}^{i}}\left(\mathbf{F}^{1}, \mathbf{F}^{2}, \mathbf{F}^{3}\right)$.

\section{Conclusion}

We proved that a repetitive lattice of elastic bars interacting by elastic moments is equivalent to a continuous medium when the number of its elementary cells is large. We obtained a definition of the equivalent medium stresses in terms of the bar tensions and of the moments between interacting bars, and we determined the equivalent medium constitutive law. This law is obtained through the solving of a lattice problem on the reference cell. Moreover, we proved that the law is frame invariant, hyperelastic, and easy to compute. This work is directly applicable to the myocardium modelling, where data on the behaviour of isolated cardiomyocytes become available. In this particular case, it will be of interest in future work to take into account the extracellular matrix which is mainly responsible for the material global incompressibility.

Acknowledgements. We wish to thank Pierre-Simon Jouk, Yves Usson and Gabrielle Michalowicz who originated this work and shared with us some of their biological knowledge during many fruitful exchanges.

\section{REFERENCES}

[1] T. Arts, R.S. Reneman and P.C. Veenstra, A model of the mechanics of the left ventricle. Ann. Biomed. Engrg. 7 (1979) 299-318.

[2] A. Bensoussan, J.L. Lions and G. Papanicolaou, Asymptotic Analysis for Periodic Structures. North-Holland, Amsterdam (1978).

[3] F. Brezzi and M. Fortin, Mixed and Hybrid Finite Element Methods, Springer Series in Computational Mathematics 15. Springer-Verlag, New York (1991).

[4] M. Briane, Three models of non periodic fibrous materials obtained by homogenization. ESAIM: M2AN 27 (1993) $759-775$.

[5] H. Cai, Loi de comportement en grandes déformations du muscle à fibres actives. Application à la mécanique du cœeur humain et à sa croissance. Thèse de l'Université de Savoie (1998).

[6] D. Caillerie and B. Cambou, Les techniques de changement d'échelles dans les milieux granulaires, in Micromécanique des milieux granulaires. Hermès Sciences, Paris (2001).

[7] R.S. Chadwick, Mechanics of the left ventricle. Biophys. J. 112 (1982) 333-339.

[8] D. Chapelle, F. Clément, F. Génot, P. Le Tallec, M. Sorine and J.M. Urquiza, A Physiologically-Based Model for the Active Cardiac Muscle Contraction, in Functional Imaging and Modeling of the Heart, Katila, Magnin, Clarysse, Montagnat and Nenonen Eds., LNCS 2230. Springer (2001) 128-133.

[9] P.G. Ciarlet, Mathematical Elasticity. Vol. 1: Three-Dimensional Elasticity. North-Holland, Amsterdam (1987).

[10] D. Cioranescu and J. Saint Jean Paulin, Homogenization of Reticulated Structures, Applied Mathematical Science 136. Springer-Verlag, New York (1999).

[11] Y.C. Fung, Biomechanics: Mechanical Properties of Living Tissues. 2nd ed., Springer-Verlag, New York (1993).

[12] M. Gurtin, An Introduction to Continuum Mechanics. Academic Press, San Diego (1981).

[13] P.S. Jouk, Y. Usson, G. Michalowicz and L. Grossi, Three-dimensional cartography of the pattern of the myofibres in the second trimester fetal human heart. Anat. Embryol. 202 (2000) 103-118.

[14] J.D. Humphrey, R.K. Strumpf and F.C.P. Yin, Determination of a constitutive relation for passive myocardium: I. A new functional form. J. Biomech. Engrg. 112 (1990) 333-339.

[15] J.D. Humphrey, R.K. Strumpf and F.C.P. Yin, Determination of a constitutive relation for passive myocardium: II. Parameter estimation. J. Biomech. Engrg. 112 (1990) 340-346.

[16] D.H.S. Lin and F.C.P. Yin, A multiaxial constitutive law for mammalian left ventricular myocardium in steady-state barium contracture or tetanus. J. Biomech. Engrg. 120 (1998) 504-517.

[17] G. Moreau and D. Caillerie, Continuum modeling of lattice structures in large displacement. Applications to buckling analysis. Comput. \& Structures 68 (1998) 181-189.

[18] A. Mourad, L. Biard, D. Caillerie, P.-S. Jouk, A. Raoult, N. Szafran and Y. Usson, Geometrical modelling of the fibre organization in the human left ventricle, in Functional Imaging and Modeling of the Heart, Katila, Magnin, Clarysse, Montagnat, Nenonen Eds., LNCS 2230. Springer (2001) 32-38.

[19] M.P. Nash and P.J. Hunter, Computational mechanics of the heart. J. Elasticity 61 (2000) 113-141.

[20] C.S. Peskin, Fiber architecture of the left ventricular wall: An asymptotic analysis. Comm. Pure Appl. Math. XLII (1989) $79-113$. 
[21] F. Pradel, Homogénéisation des milieux continus et discrets périodiques orientés. Thèse de l'École Nationale des Ponts et Chaussées (1998).

[22] E. Sanchez-Palencia, Non Homogeneous Media and Vibration Theory, Monographs in Physics 127. Springer-Verlag, Berlin (1980).

[23] D.D. Streeter, Gross morphology and fiber geometry of the heart, in Handbook of Physiology. The cardiovascular system, R.M. Berne, N. Sperelakis and S.R. Geiger Eds., Am. Phys. Soc. Williams \& Wilkins, Baltimore (1979).

[24] L.A. Taber and R. Perucchio, Modeling heart development. J. Elasticity 61 (2000) 165-197.

[25] H. Tollenaere and D. Caillerie, Continuous modeling of lattice structures by homogenization. Adv. Engrg. Software 29 (1998) 699-705.

[26] C. Truesdell, A First Course in Rational Continuum Mechanics. Academic Press, New York (1977).

[27] T.P. Usyk, R. Mazhari and A.D. McCulloch, Effect of laminar orthotropic myofiber architecture on regional stress and strain in the canine left ventricle. J. Elasticity 61 (2000) 143-165.

[28] K. Washizu, Variational Methods in Elasticity and Plasticity. 2nd ed., Pergamon Press (1975).

[29] F.C.P. Yin, R.K. Strumpf, P.H. Chew and S.L. Zeger, Quantification of the mechanical properties of noncontracting canine myocardium under simultaneous biaxial loading. J. Biomech. 20 (1987) 577-589.

[30] M. Zile, M.K. Cowles, J.M. Buckley, K. Richardson, B.A. Cowles, C.F. Baicu, G. Cooper IV abd V. Gharpuray, Gel stretch method: a new method to measure constitutive properties of cardiac muscle cells. Am. J. Physiol. 274 (1998) H2188-2202.

To access this journal online:

www.edpsciences.org 